\title{
X-ray Spectra of Circumgalactic Medium Around Star-Forming Galaxies: Connecting Simulations to Observations
}

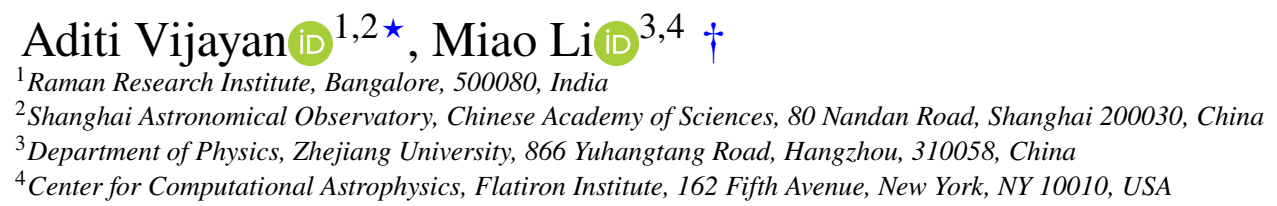

Accepted XXX. Received YYY; in original form ZZZ

\begin{abstract}
The hot component of the circum-galactic medium (CGM) around star forming galaxies is detected as diffuse X-ray emission. The X-ray spectra from the CGM depend on the temperature and metallicity of the emitting plasma, providing important information about the feeding and feedback of the galaxy. The observed spectra are commonly fitted using simple 1-Temperature (1-T) or 2-T models. However, the actual temperature distribution of the gas can be complex because of the interaction between galactic outflows and halo gas. Here we demonstrate this by analysing 3-D hydrodynamical simulations of the CGM with a realistic outflow model. We investigate the physical properties of the simulated hot CGM, which shows a broad distribution in density, temperature, and metallicity. By constructing and fitting the simulated spectra, we show that, while the 1-T and 2-T models are able to fit the synthesized spectra reasonably well, the inferred temperature(s) does not bear much physical meaning. Instead, we propose a log-normal distribution as a more physical model. The log-normal model better fits the simulated spectra while reproducing the gas temperature distribution. We also show that when the star formation rate is high, the spectra inside the bi-conical outflows are distinct from that outside, as outflows are generally hotter and more metal-enriched. Finally, we produce mock spectra for future missions with the eV-level spectral resolution, such as Athena, Lynx, HUBS and XRISM.
\end{abstract}

Key words: galaxies:evolution, spiral, haloes - X-rays:galaxies - hydrodynamics - methods: numerical

\section{INTRODUCTION}

Circumgalactic medium (CGM), the multiphase gas surrounding a galaxy, has a symbiotic relationship with the galaxy. CGM serves as a reservoir providing fuel for star formation (e.g. Lehner et al. 2012, 2013; Ford et al. 2014), and the feedback from the galaxy leaves imprints on the CGM (Oppenheimer \& Davé 2008; Hummels et al. 2013; Fielding et al. 2017; Suresh et al. 2017; Li \& Tonnesen 2020; Davies et al. 2020). The CGM thus plays a critical role in galaxy evolution.

The X-ray emitting hot phase of the CGM, with a temperature $\gtrsim 10^{6} \mathrm{~K}$, is also referred to as "galactic corona". X-ray observations have revealed a connection between the CGM and the galaxies hosting it. For star-forming galaxies, the X-ray luminosities of the hot CGM have positive scaling relations with the galaxy's mass and its star formation rate (Strickland et al. 2004; Tüllmann et al. 2006; Li \& Wang 2013; Wang et al. 2016). Further, the X-ray emission is

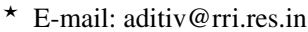

$\dagger$ miaoli@zju.edu.cn spatially correlated with star forming regions (Grimes et al. 2005; Owen \& Warwick 2009). The extended X-ray emission has been detected up to several $\sim 10 \mathrm{~s} \mathrm{kpc} \mathrm{in} \mathrm{massive} \mathrm{spirals} \mathrm{(Yamasaki} \mathrm{et} \mathrm{al.}$ 2009; Anderson \& Bregman 2011; Dai et al. 2012; Anderson et al. 2016; Bogdán et al. 2013a,b, 2017; Lopez et al. 2020). Around early type galaxies, the hot halos have been detected out to $\sim 10 \mathrm{spc}$ and the X-ray luminosity is correlated with the optical luminosity (Forman et al. 1985; O'Sullivan et al. 2001; Mulchaey \& Jeltema 2010).

Theoretically, galaxy coronae can develop via two processescosmic accretion and galactic feedback. Gas infall into the dark matter (DM) halo from the inter-galactic medium (IGM) creates virial shocks, heating the gas to the virial temperature of the galaxy. If the halo is sufficiently massive $\left(\mathrm{M}_{\mathrm{vir}} \gtrsim 10^{12} \mathrm{M}_{\odot}\right)$, the shockheated gas has a very long cooling time (e.g., $\sim 6 \mathrm{Gyr}$ for a $10^{7} \mathrm{~K}$, $10^{-4} \mathrm{~cm}^{-3}$ gas and assuming cooling rate of $\sim 10^{-23} \mathrm{erg} \mathrm{s}^{-1} \mathrm{~cm}^{-3}$ ) and it forms a hot CGM that is in quasi-hydrostatic equilibrium with the gravitational potential of the DM halo (McKee \& Ostriker 1977; Silk 1977; Dekel et al. 2009; Kereš et al. 2005). Feedback 
from star formation and supermassive black holes releases copious of energy which can heat and accelerate the ambient inter-stellar medium (ISM) (Fabian 2010). A fraction of the feedback energy and material can escape from the galaxy into the CGM, via galactic winds (Sarkar et al. 2015; Li et al. 2017b; Armillotta et al. 2017; Kim et al. 2017; Vijayan et al. 2018; Li \& Tonnesen 2020).

Galactic coronae are often studied through their X-ray emission spectra. The overall shape and the emission lines (if resolvable) are determined by the underlying gas properties, such as temperature, density, metallicity, etc. This information is then used to infer the galaxy formation process, such as gas feeding and feedback. It is thus critical to extract the gas properties from the spectra in a meaningful way. While interpreting the observed spectra, simple models for the temperature distribution of the underlying plasma are commonly assumed e.g., a 1-Temperature (1-T) or 2-T distribution. However, such assumptions for the CGM have rarely been been evaluated critically. In fact, there is reason to believe that the situation is more complex. Near the disk, where most of the emission arises, the hot galactic outflows undergo expansion, radiative cooling, interaction with pre-existing halo gas and other gas phases, etc. Thus, a 1-T or 2-T model may not be sufficient to capture the real temperature distribution of the gas.

In this paper, we evaluate the gas models used for interpreting the diffuse X-ray spectra from the CGM of a star forming galaxy. We use hydrodynamic simulations by Li \& Tonnesen (2020) (referred to as "L20" hereafter), which model galactic outflows and their impact on the CGM for a Milky Way-mass galaxy. The simulations of L20 are suitable for X-ray studies because they incorporate a careful treatment of hot outflows taken from the results from small-box simulations of supernovae-driven outflows from the ISM (Li et al. 2017a; Li \& Bryan 2020). We examine the X-ray emitting gas from the simulation and investigate the distribution of its temperature. We then construct synthesized X-ray spectra, and use them to evaluate the commonly used 1-T and 2-T models. We also make mock spectra for the upcoming X-ray satellites, Athena, Lynx, and HUBS. These telescopes will have unprecedented spectral resolution and sensitivity, which will transform our understanding about the CGM and thus galaxy formation.

The paper is organised as follows. In Section 2 we discuss the general setup of simulations from L20 and in Section 3 we discuss the results from the simulations, including the outflow properties of the simulations (Section 3.1), the density and temperature distributions (Section 3.2), and the X-ray emission (Section 3.3). In Section 3.4 we produce synthesized spectra from the simulation data and compare it with 1-T and 2-T models. In Section 4, we propose a new model of temperature distribution and produce spectra from this model and compare with simulations. We then discuss spatial dependence of the emission spectra in Section 5. We conclude by discussing this work with respect to previous works in Section 6, and providing mock spectra for Chandra, Athena, Lynx and HUBS by convolving simulation spectra with instrument responses (Section 6.1). We summarize this paper in Section 7.

\section{SIMULATIONS}

In this Section, we briefly discuss the simulation setup of and results from L20, data from which are used for analysis for this paper. L20 uses 3D hydrodynamical simulations to investigate how supernovae (SNe)-driven hot outflows regulate the CGM around a Milky Waymass galaxy. The outflow model - in particular, the outflow rate of mass, energy, and metals - is taken from kpc-box simulations with pc-scale resolutions that model the launching of outflows by $\mathrm{SNe}$ for various star formation surface densities (Li et al. 2017b). The hot outflow properties show a very good agreement among small-box simulations of supernovae-driven outflows which utilized different hydro-codes (Li \& Bryan 2020). Outflows driven from the ISM is multiphase, and the hot phase has $T \sim 10^{6-7} \mathrm{~K}$. For L20, only the hot phase of the outflows is added from small-box simulations because the hot phase is much more powerful than the cool phase, and can travel to distances $\gtrsim 100 \mathrm{kpc}$ away from the galaxy (see discussions in (Li \& Bryan 2020)). In contrast, most cool outflows can only travel to a few kpc (Li et al. 2017b; Kim et al. 2017; Vijayan et al. 2018; Li \& Bryan 2020).

For a detailed description of the simulation setup, one is referred to L20. Here is a brief summary: The simulation domain is $[800 \mathrm{kpc}]^{3}$ containing the galaxy at its centre. The galaxy disk in the $x-y$ plane. The gravitational potential is determined by a Burkert (1995) profile for the dark matter (DM) halo of $10^{12} M_{\odot}$, a Plummer-Kuzmin profile (Miyamoto \& Nagai 1975) for stellar disk and a spherical Hernquist (1993) profile for the bulge. The spatial resolution is progressively finer near the center, with 0.39 $\mathrm{kpc}$ in the inner $[50 \mathrm{kpc}]^{3}, 0.78 \mathrm{kpc}$ in the $[100 \mathrm{kpc}]^{3}$, and so forth. The Grackle library (Smith et al. 2017) is used to calculate the metallicity-dependent gas cooling. The reader is directed to Section 3.1 of L20 for further details on cooling.

The initial gas distribution is in overall hydrostatic equilibrium with the DM potential with an inner core at radii $<40 \mathrm{kpc}$ (see Figure 1 of L20 and is overwritten by subsequent gas evolution. The initial gas distribution does not include cold gas as gas at this temperature has minuscule contribution to the X-ray emission, which is the primary focus of this paper. The gas has a uniform temperature of $10^{6} \mathrm{~K}$ i.e. around the virial temperature of Milky Way (MW) halo, and a low metallicity $Z=0.2 Z_{\odot}$, where $Z_{\odot} \equiv 0.01295$. The total gas mass is a free parameter, represented by $\mathrm{n}_{0}$, the mean number density within $R=200 \mathrm{kpc}$. The fiducial $n_{0}$ is determined by comparing $L_{X}$ of the resulted CGM to the observations with the same stellar mass and star formation rate (SFR).

The outflows are parameterised using the mass, metal and energy loading factors multiplied by the designated SFR (see Equations 7, 8 and 9 of L20). The outflows from star formation episodes are injected in a small region above and below the disk at a height of $3 \mathrm{kpc}$ (no cool gaseous disk is present in the simulation as initially). Temporally, each star formation event occurs every $\Delta t \simeq 9.9 \mathrm{Myr}$ with a randomly chosen $x-y$ location. The added outflows are metalenriched and initially overpressured, and they expand subsequently and interact with the ambient gas.

In this paper, we analyse the results from two simulations with different parameterizations. One is when star formation (SF) is relatively quiescent and widespread on the disk, like the current state of the MW. The other is where SF is more intense and centrally concentrated. The different cases have SFRs of 3 and $10 \mathrm{M}_{\odot}$ $\mathrm{yr}^{-1}$, and $\dot{\Sigma}_{\mathrm{SFR}}$ of $6 \times 10^{-3}$ and $0.3 \mathrm{M}_{\odot} \mathrm{kpc}^{-2} \mathrm{yr}^{-1}$, respectively. From the small-box simulations, such $\dot{\Sigma}_{\text {SFR }}$ correspond to mass, energy, and metal loading factors of hot outflows [1.0, 0.3,0.5] and $[0.2,1.0,0.5]$, respectively. In the former case, the specific energy of hot outflows is lower and outflows cannot escape from the DM halo of $10^{12} M_{\odot}$, therefore they form a large-scale fountain around the galaxy. In the latter, hot outflows are powerful enough to escape the halo and form a bipolar structure ( $\mathrm{Li} \&$ Tonnesen, in prep). In both cases, cool CGM gas precipitates out of the hot atmosphere at later times. We denote the two different runs as SFR3 and SFR10. The X-ray luminosity $L_{X}(0.5-2.0 \mathrm{keV})$ of the CGM for the two cases are $8 \times 10^{39}$ and $2 \times 10^{40} \mathrm{erg} \mathrm{s}^{-1}$, respectively. 


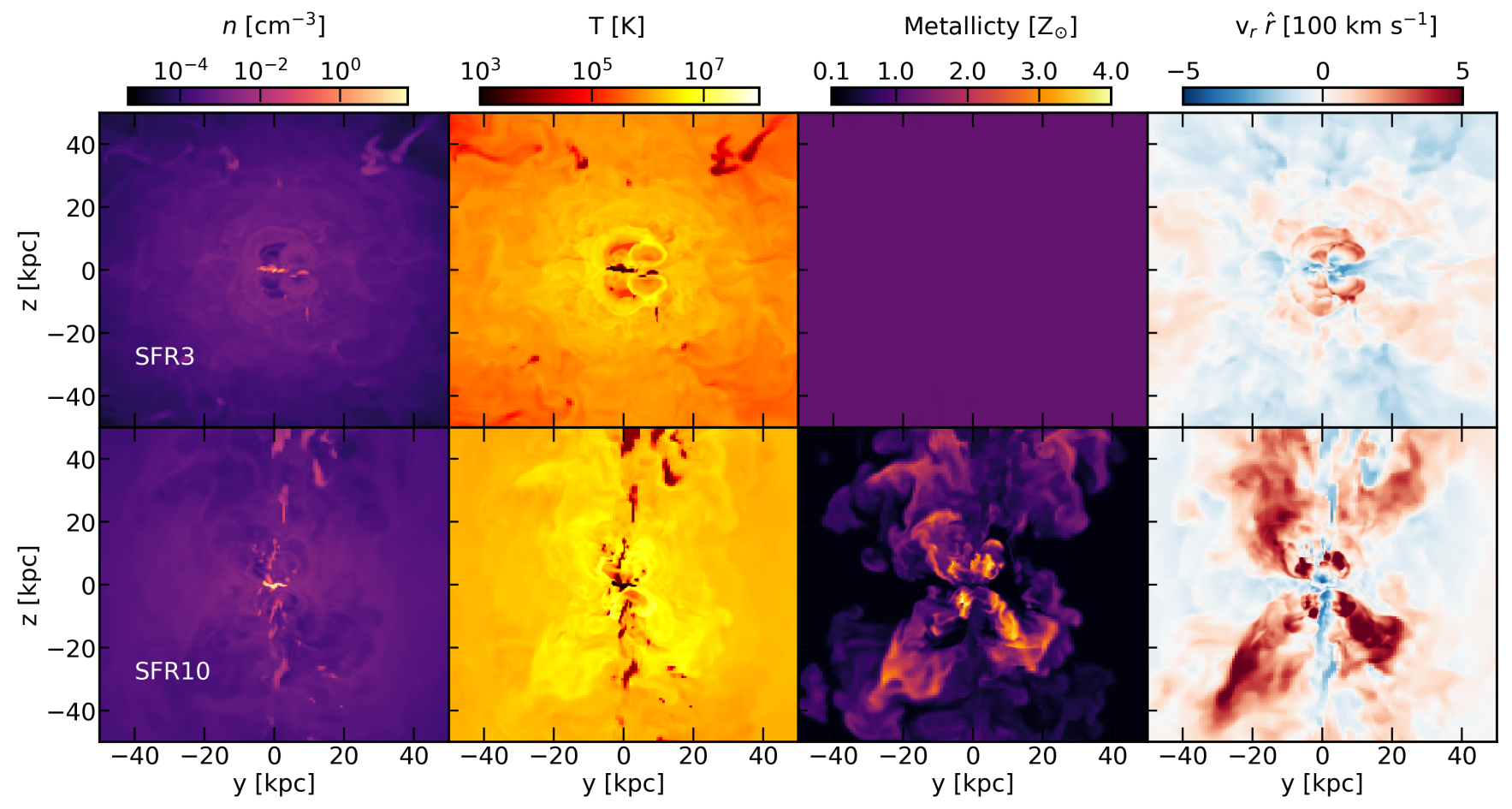

Figure 1. An edge view for $x=0$ slices from the simulations of Li \& Tonnesen (2020) for SFR3 (top) and SFR10 (bottom) runs at 3 and 1.3 Gyr, respectively, showing density, temperature, metallicity, and radial velocity. SFR3 forms a large-scale fountain flows, while SFR10 has bi-conical outflows. Gas near the disk show a wide range of density and temperature, as a result of episodic outflows.

Most of the soft $L_{X}$ of the CGM comes from $\lesssim[30 \mathrm{kpc}]^{3}$, where density is highest (as shown in Figure 3 below). Consequently, our analysis is limited to the inner $[50 \mathrm{kpc}]^{3}$ of the simulation box. We analyse the simulation data for these runs at times after $L_{X}$ has reached a steady state. We show the time evolution of the results in Appendix A. In Section 6, we briefly discuss our results in the context of other simulations.

\section{RESULTS}

\subsection{Visualization of Simulated CGM}

We first discuss the distribution of various physical quantities within a radius of $50 \mathrm{kpc}$ of the simulation box.

In Figure 1, we show $x=0$ slices (galaxy disk viewed edgeon) of density, temperature, metallicity and radial-velocity for SFR3 (top) and SFR10 (bottom). The snapshots are taken at $3 \mathrm{Gyr}$ and 1.3 Gyr for SFR3 and SFR10, respectively. At these time steps the outflows have significantly changed the initial conditions of the halo gas and the CGM has reached a quasi-steady state (see Appendix A for a discussion).

Fast and hot outflows emerge from each star formation episode, disrupting the initial isothermal gas profile. In SFR3 these episodes result in formation of expanding bubbles, distinctly visible as nearly spherical regions with low densities and high temperatures (left two panels on top).

In SFR3, at smaller radii ( $\mathrm{R} \lesssim 10 \mathrm{kpc}$ ), low-density, hightemperature bubbles expand into the surrounding CGM. Every outflow injection event drives an outward-moving shock into the ambient medium. Beyond the outer-shock, the density and temperature profiles decrease with radius. Gas which cools at large radii loses pressure support and falls under gravity. We can clearly see this cooled gas as a high-density $\left(\sim 1 \mathrm{~cm}^{-3}\right)$ and low-temperature feature $\left(\sim 10^{4} \mathrm{~K}\right)$ feature in the top panel of Figure 1. Eventually, this gas settles at the bottom of the potential well, that is, at the centre of the box. Due to a long period of star formation, the metallicity of the ambient medium has been completely overwritten by gas with a higher metallicity around $\sim 1.2 \mathrm{Z}_{\odot}$. The spherical outflows have expanded in all the directions and produced uniform metallicity in the region. The outflowing gas has velocities of a few $\sim 100 \mathrm{sm} \mathrm{s}^{-1}$. For SFR3, the hot outflows do not possess sufficiently large velocities. At large radii, these outflows turn around and form large-scale fountain flows.

Outflows in SFR10 have a bi-conical shape because they have a specific energy larger than the halo potential, and are able to break out from the halo. At the launching site, the outflows are hotter than the ambient medium. As the injected gas progresses into the CGM, it mixes with the pre-existing hot gas, creating a wide distribution in density as well as in temperature. ${ }^{1}$ The cold and dense gas seen in the slices is produced by the precipitation of the CGM as a result of the interaction between hot outflows and pre-existing halo gas. The gas within the $50 \mathrm{kpc}$ radius show metallicity values over a wide range. The most enriched part, at $\sim 4 \mathrm{Z}_{\odot}$, is the newly injected hot outflows. Lower-metallicity gas is created as a result of mixing between these super- $\mathrm{Z}_{\odot}$ outflows and the low-metallicity ambient medium. In the bipolar region, velocities are large and

\footnotetext{
1 Note that such a mixing process between "hot" and "hotter" material is different from mixing occurring between $10^{4}$ and $10^{6} \mathrm{~K}$, which arises when cold gas is entrained by hot outflows and produces cooler, mainly UV-emitting gas (Suchkov et al. 1996; Fielding \& Bryan 2021; Nguyen \& Thompson 2021).
} 


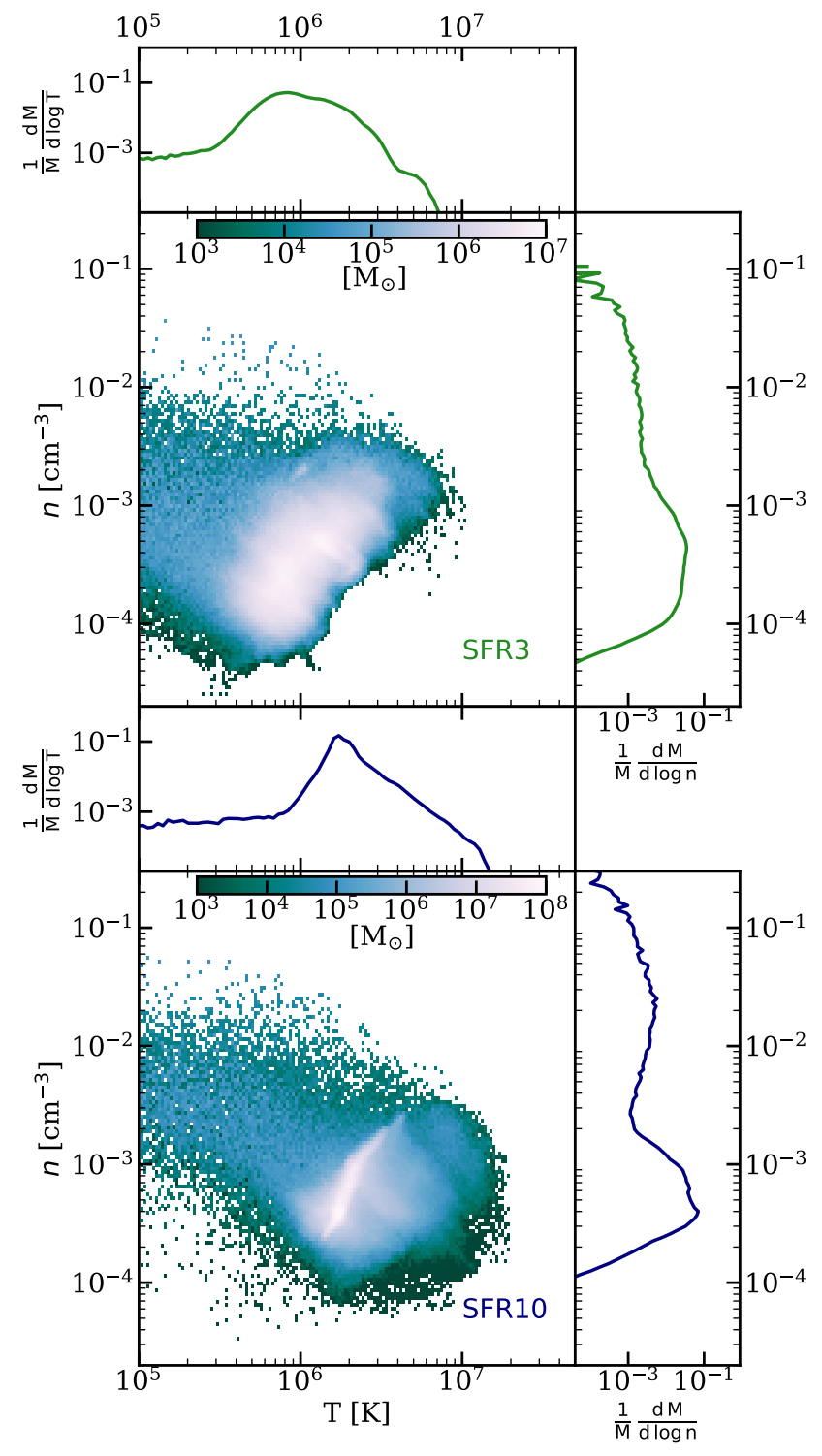

Figure 2. Mass distribution of density and temperature distribution for SFR3 (top) and SFR10 (bottom) for the region of interest ( essentially a sphere of radius $50 \mathrm{kpc}$ from which we remove a cylinder, $\left.\pi 20 \times 20 \times( \pm 3) \mathrm{kpc}^{3}\right)$. The snapshots are at $\mathrm{t}=3$ and $1.3 \mathrm{Gyr}$, respectively. The colour shows mass in each density and temperature bin. The side panels show mass distribution of density (right) and temperature (top). Both runs show that hot gas has a broad distribution of density and temperature.

predominantly outward, showing that outflows are powerful enough to break out through the pre-existing gas.

\subsection{Histograms of Density and Temperature}

The temperature and density of the hot gas are critical in determining the X-ray spectra. In this Section, we will discuss in detail the density and temperature distribution of the simulated CGM.

For the analysis of hot gas, we have removed the "galaxy" region, from a sphere of radius $50 \mathrm{kpc}$, due to the proximity to the injection region. The removed region is a cylinder with its axis along the $z$-axis, $\pi 20 \times 20 \times( \pm 3) \mathrm{kpc}^{3}$. In X-ray observations of diffuse gas in CGM, the region near the disk is also removed in

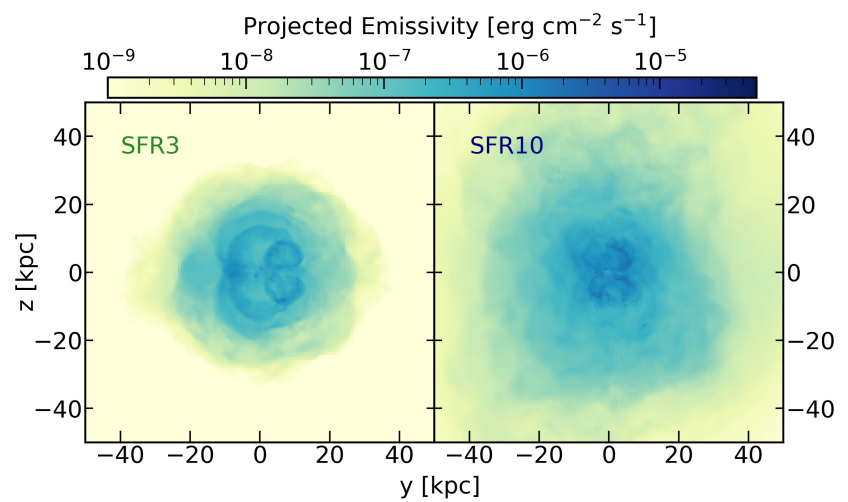

Figure 3. Projected emissivity at $0.5-2.0 \mathrm{keV}$ for SFR3 (left) and SFR10 (right) runs.In SFR3 case, emission is most significant for radii $\lesssim 20 \mathrm{kpc}$, while for SFR10 the emission is more extended. The bi-conical structure of SFR10 is not very prominent in the projected emission map because the emission is dominated by gas which lies outside the outflow cones.

order to include only contribution from the extra-planar region and to remove contribution from point X-ray sources. We will refer to the remaining region, i.e. total box minus the galaxy, as the "region of interest".

In Figure 2, we show the mass distribution of density and temperature for the regions of interest of the two runs. They are taken at the same snapshots as Figure 1. The colour indicates mass per density and temperature bin. It is clear that in both runs, the gas occupies a wide range in density and temperature.

The side panels of Figure 2 show 1-D fractional mass distribution of density (right) and temperature (top) of the gas in the region of interest. Despite morphological differences in the two runs (see Figure 1), the 1-D profiles are qualitatively similar- the X-ray emitting gas shows broad distributions around a single peak. The temperature profile is smoother and broader for SFR3. In SFR10, the peak of the 1-D temperature distribution is sharper. Around this peak is the gas temperature of the ambient medium outside the bi-conical region, which has not been largely affected by the outflows.

The mass distribution presented in this section represents a snapshot in the simulation. However, we note that these times are after the systems have reached a quasi-steady state and the distributions do not change thereafter. We show the temporal variations in the mass distribution in the Appendix A.

\subsection{X-ray Emission}

Figure 3 shows the edge-on view of the projected emissivity at $0.5-2 \mathrm{keV}$ for the two runs. We obtain X-ray emissivity using the add_xray_emissivity_field module of yt with the APEC emission tables ${ }^{2}$. Firstly, SFR10 has a more extensive and brighter emission than SFR3. For SFR3, the high-emissivity regions lie along the shells corresponding to recent outbursts. Though the hot outflows have expanded to fill the entire region of interest, the gas beyond the radius of $\sim 20 \mathrm{kpc}$ has lower densities as well as lower temperatures and does not emit as strongly in X-rays. In SFR10, the hot ( $\gtrsim 10^{6}$ $\mathrm{K})$ gas also fills the entire region of interest. Though, the bi-conical feature of the outflows is not very prominent on the emissivity map

2 APEC v 2.0.2 downloaded from https://yt-project.org/data/. 


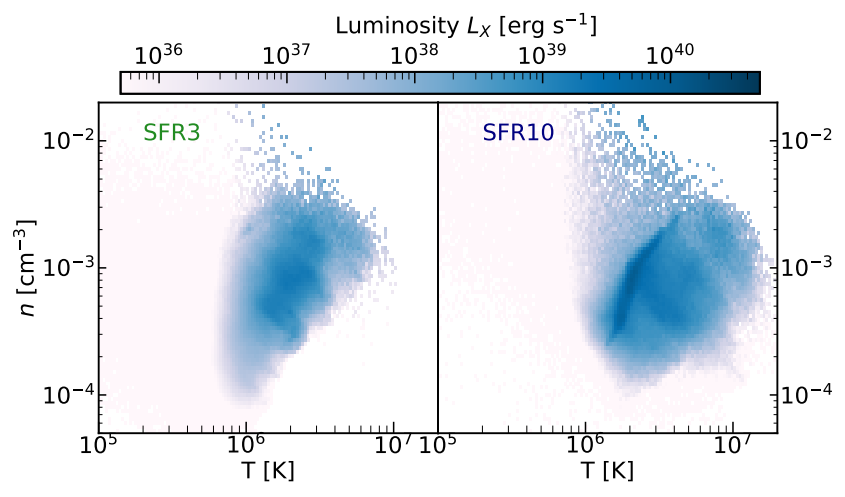

Figure 4. X-ray luminosity $L_{X}$ as a function of density and temperature. This is similar to Figure 2 but the colour bar represents $L_{X}(0.5-2.0 \mathrm{keV})$. The luminosity distribution closely follows the mass distribution as shown in Figure 2.

because the emission is dominated by the gas lying outside of the cone.

In Figure 4, we show the distribution of X-ray luminosity $L_{X}$ $(0.5-2.0 \mathrm{keV})$ as a function of density and temperature for the regions of interest. The distribution of X-ray luminosity closely follows that of the mass, as shown in Figure 2. This is not surprising because luminosity is proportional to the gas mass. Gas with densities of $10^{-4}-10^{-3} \mathrm{~cm}^{-3}$ and temperatures of one to a few $10^{6} \mathrm{~K}$ dominates the X-ray emission. For SFR3, the emission is dominated by gas with temperature $\gtrsim 10^{6} \mathrm{~K}$, even though significant mass lies near the peak of the cooling curve, i.e., $10^{5-6} \mathrm{~K}$. This is expected as $10^{5-6} \mathrm{~K}$ gas emits mostly in UV bands.

\subsubsection{Spectra Generation}

In this Section, we describe how we construct the spectra from the simulated CGM. We use the python-based module pyXSIM ${ }^{3}$, an implementation of PHOX (Biffi et al. 2012, 2013). pyXSIM generates X-ray photons in each cell of the simulation domain using the gas density, temperature and metallicity and an X-ray emission model based on the APEC tables which assume collisional ionization equilibrium $(\mathrm{CIE})^{4}$. The total number of photons generated also depends on input parameters such as exposure time (100 ks), effective area $\left(1000 \mathrm{~cm}^{2}\right)$, and the distance of the source $(1 \mathrm{Mpc})$. We have chosen arbitrarily large values for effective area and exposure time in order to generate sufficient number of photons for the next steps. The coordinates and distance of the source for the spectra discussed in this Section are also arbitrary. From this collection of photons, a subset is projected along the line-of-sight and a Galactic absorption model is applied, assuming a hydrogen column density of $1.93 \times 10^{20} \mathrm{~cm}^{-2}$. We use the Wisconsin Absorption Model ("wabs", Morrison \& McCammon 1983) and generate spectra in the X-ray range $0.5-2.0 \mathrm{keV}$. We note that the spectra shown are not convoluted with any instrument response.

Using this procedure we produce the spectra for SFR3 and SFR10 and present them in the topmost and the fifth panels of Figure 5, respectively. In the left panel we show the low-resolution

\footnotetext{
3 http://hea-www.cfa.harvard.edu/ jzuhone/pyxsim/index.html.

4 The timescale to achieve CIE is $\sim 10^{4} \mathrm{yr}$, which is much shorter than both dynamical and cooling time scales of the gas.
}

Table 1. Best-fit parameters for the simulated spectra

\begin{tabular}{|l|l|l|}
\hline Parameter & SFR3 & SFR10 \\
\hline $\mathrm{T}_{1 \mathrm{~T}}[\mathrm{MK}]$ & 2.2 & 3.8 \\
\hline $\mathrm{T}_{2 \mathrm{~T}, \text { low }}[\mathrm{MK}]$ & 0.8 & 1.7 \\
\hline $\mathrm{T}_{2 \mathrm{~T}, \text { high }}[\mathrm{MK}]$ & 2.8 & 3.0 \\
\hline $\mathrm{Z}\left[\mathrm{Z}_{\odot}\right]$ & 1.19 & 0.58 \\
\hline$\mu_{T}[\mathrm{MK}]$ & 1.0 & 2.2 \\
\hline $\log \left(\sigma_{\mathrm{T}}\right)$ & 0.51 & 0.46 \\
\hline
\end{tabular}

spectra which is binned at $130 \mathrm{eV}^{5}$. The right panel shows the highresolution spectra, binned at $1 \mathrm{eV}$. The differences in the temperature distribution in the two runs are reflected in their respective spectra. Because of the lower temperature of the gas present in SFR3, its spectra is steeper than that of SFR10.

\subsection{1-T and 2-T Models}

In this Section we introduce the 1-T and 2-T models for fitting X-ray spectra from observations. We will then compare the spectra generated using these models with those generated using the simulation data.

The 1-T and 2-T models of plasma emission are commonly used to fit the observed X-ray spectra. The 1-T model assumes that the $\mathrm{X}$-ray emitting gas is at a single temperature, while the 2-T model assumes that the observed spectra is results from a superposition of spectra at two different temperatures.

\subsubsection{Spectra from 1-T Model}

To construct the 1-T model, we generate a "uniform box", a yt object having identical density and temperature values in all cells. To keep the model simple, we set its metallicity as the average metallicity of the gas in the simulation. To get the best fit parameters for temperature, we adopt a "blind-search" method. We use the spectra from simulation as the "ground truth" and generate multiple spectra with varying temperatures of the uniform box. To determine the best-fit spectrum for a run, for each high resolution spectrum from the uniform box we calculate the net fractional difference (NFD) which is given by $\sqrt{\Sigma\left((\text { Simulation }- \text { Model })^{2} / \text { Simulation }^{2}\right)}$.

We note that the quantity we define as NFD is different from the conventional "reduced chi square", the standard statistic used by observers to determine the goodness of the fit, given by $\chi_{\text {reduced }}^{2} \equiv \Sigma_{i} \frac{\left(O_{i}-C_{i}\right)^{2}}{v \sigma_{i}^{2}}$, where $O_{i}, C_{i}, \sigma_{i}^{2}$ and $v$ are the observations, the model and the variance, and the degrees of freedom of the data, respectively. We stress here that for our purpose the NFD defined by us suits well. We select the temperature for which the NFD is minimum, indicated in Table 1. Once the best-fit temperature is set, we adjust the normalisation of the spectra to minimise the NFD again. The values of NFD for the best-fit temperature and normalisations are indicated in Figure 6.

We represent the best-fit 1-T spectra using $\Delta$, which is the fractional difference between the simulation spectra and the bestfit spectra at a given energy. $\Delta$ is given by $\Delta=$ (Simulation Model)/Simulation and it characterises the deviation of the model from the simulation. The second and sixth panels of Figure 5 show the $\Delta$ for 1-T model for SFR3 and SFR10, respectively. For SFR3,

5 Corresponds to the resolution of Chandra ACIS in the wide field imaging mode- https://cxc.harvard.edu/cal/Acis/. 
$\Delta$ becomes negative in the higher energy range as the model overpredicts emission from the CGM. Because the model is biased towards higher temperatures, for SFR10, $\Delta$ is large in the lower energy range, while it is close to zero for higher energies. Despite its simplicity, the spectra from the 1-T model fits the simulation spectra well for both the runs. The models fits the spectra especially well for high energy range $(\gtrsim 1.0 \mathrm{keV})$. We note that for low-resolution spectra (left panels) it appears that 1-T model is a close fit.

In Figure 7, we depict a visualization of the 1-T model. In top and bottom panels, the solid curves show the fractional mass distribution of the logarithmic temperature for the simulations, which are identical to the top panels of Figure 2. The best-fit 1-T models are represented by dashed vertical line in the Figure.

From Figure 5, we conclude that 1-T model is able to match the spectra well. However, the mass distribution represented by the 1-T model is drastically different from the underlying temperature distribution of the gas, which is quite broad. Thus, we conclude that a 1-T model, while being able to reproduce the spectra, cannot faithfully represent the physical temperatures in the CGM. 


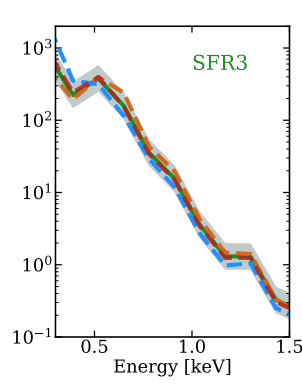

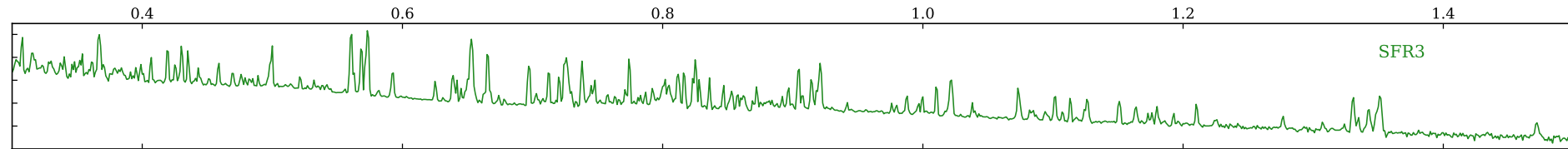

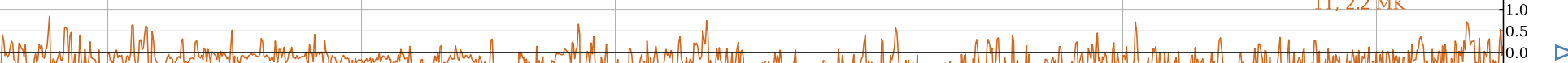

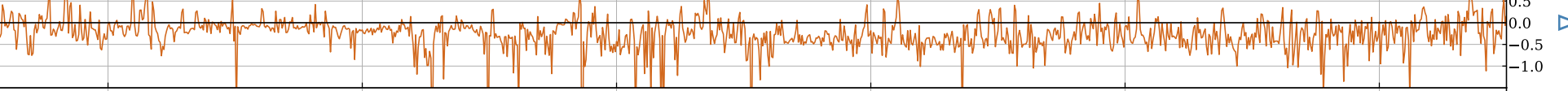

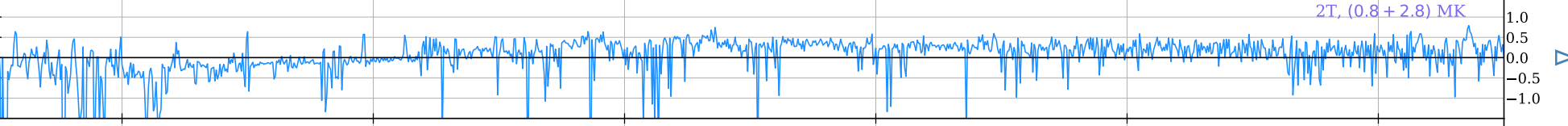

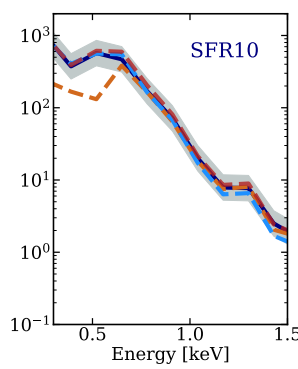

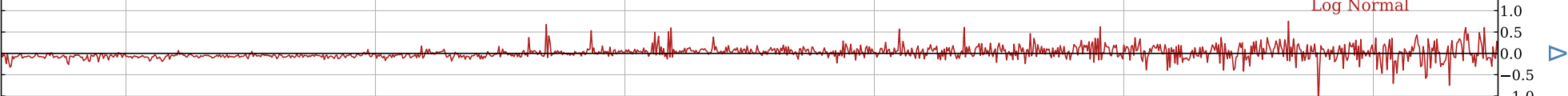

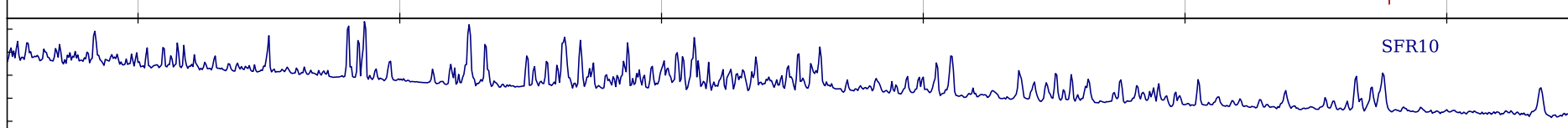

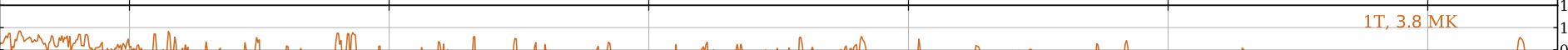
$1 \mathrm{~T}, 3.8 \mathrm{MK}$

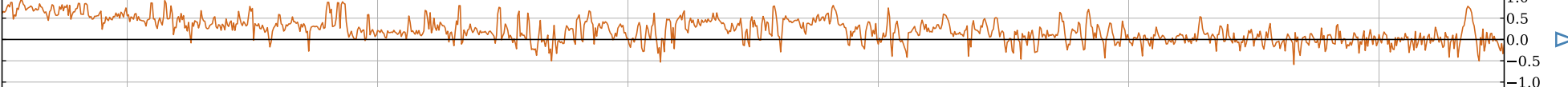
(1.7)

Figure 5. Spectra from simulations compared with best-fit spectra from different models. Spectra on the left are binned at $130 \mathrm{eV}$, similar to the Chandra resolution, while those on the right are binned at $1 \mathrm{eV}$. Solid lines represent the spectra from the simulations of SFR3 (green) and SFR 10 (blue) at 3.0 Gyr and 1.3 Gyr respectively. In the left column, the best-fit spectra from 1-T, 2-T and the log-normal models are represented by orange, blue and red curves, respectively. In the right column panels, we compare the high resolution spectra from simulations with the corresponding $\Delta$ values from the different models- 1-T (orange curves in second and sixth panels), 2T (blue curves in third panel from top and second panel from the bottom), and log-normal (red curves in fourth panel from top and the last panel). The grey shaded region in the left panels indicates the temporal variations in the spectra over the duration of the simulation. 


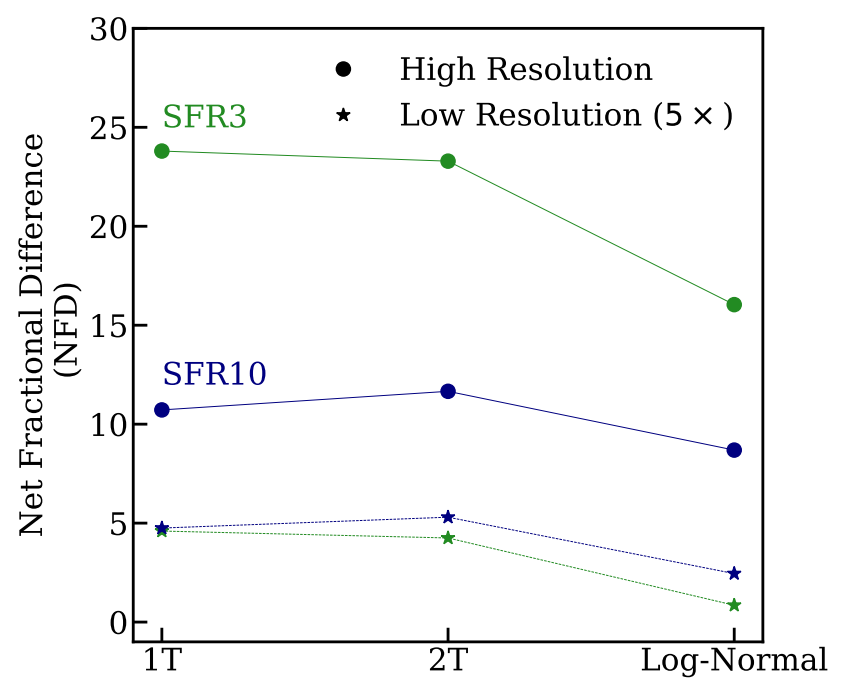

$\begin{array}{llllll}\text { Figure } & \text { 6. } & \text { Comparison } & \text { of } & \text { NFD } & \equiv \\ \sqrt{\Sigma\left((\text { Simulation }- \text { Model })^{2} / \text { Simulation }^{2}\right)} & \text { among } & 1-T, & 2-T & \text { and } & \text { Log- }\end{array}$ Normal models. Circles and stars represent SFR3 and SFR10, respectively. We have multiplied the NFD values for the low-resolution spectra by a factor of 5 for clarity. The log-normal model leads to a better fit (smaller NFD than the 1-T/2-T models. We point out here that the number of bins used for high- and low- resolution spectra are different and thus, the curves for high- and low-resolutions should not be compared.

\subsubsection{Spectra from 2-T Model}

When fitting for the observed data, if a 1-T model cannot reproduce the spectra, a 2-T model is usually invoked, which adds an additional temperature component. Three parameters determine the shape of the spectra in the 2-T model, viz., the two temperatures and the ratio of their normalisations. However, it is possible that more than one combinations of these parameters can fit the same data reasonably well. Instead of exploring a full parameter space, we here discuss one possible way of finding the best-fits for these parameters for the simulation spectra. Our procedure for fitting the spectra differs from the way spectra are fit by observers. While we fix one temperature and iterate to find the second temperature, traditionally observers pursue to fit the two temperatures simultaneously and minimize the standard reduced chi-squared statistic.

Following the method described in Section 3.4.1 for the 1-T model, we now construct two separate uniform boxes, each representing one of the two temperatures of the 2-T model. For simplicity, we use $\mathrm{T}_{\text {peak }}$, the temperature at which the mass-weighted temperature distributions (see Figure 2) peak, as the lower temperature for this model denoted as $\mathrm{T}_{2 \mathrm{~T}}$,low. Next, starting from a guess value for the higher temperature, $T_{2 T}$, high, we calculate NFD for a range of temperatures. As previously, we obtain the best match to the simulations spectra by selecting the $\mathrm{T}_{2 \mathrm{~T}}$, high with the smallest NFD. We indicate $T_{2 T}$, low and $T_{2 T}$, high for the two runs in Table 1 . We then adjust the normalisations of the two uniform boxes to minimize NFD.

We obtain the spectra from the uniform boxes at $\mathrm{T}_{2 \mathrm{~T} \text {, low }}$ and $\mathrm{T}_{2 \mathrm{~T}}$, high and the total 2-T spectra is obtained by summing their normalised values. In Figure 5, we show the 2-T spectra binned at at $130 \mathrm{eV}$ (left) and $1 \mathrm{eV}$ (right). As previously, we show how $\Delta$ between the simulated and the best-fit 2-T spectra in the third from top and second from bottom for SFR3 and SFR10, respectively. The

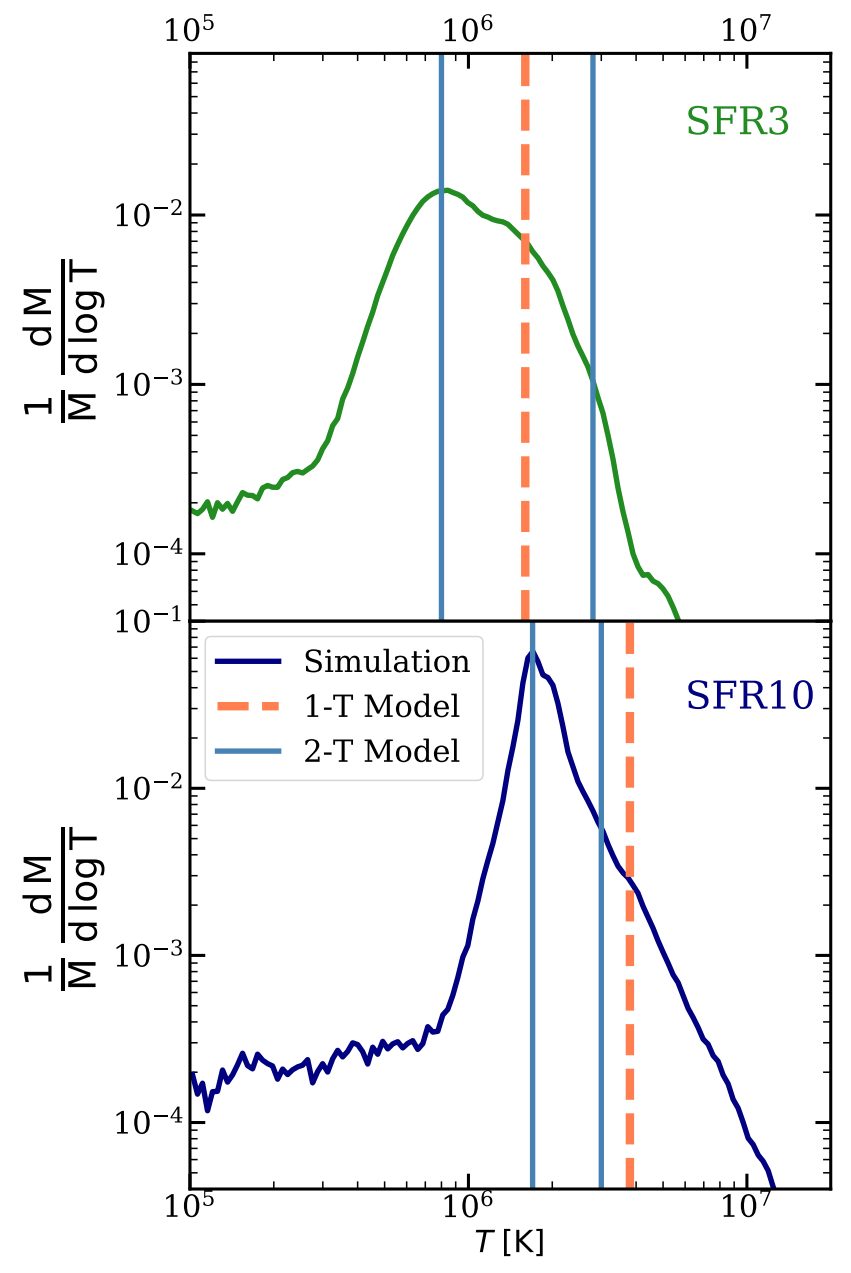

Figure 7. Comparison of the mass distribution of the temperature between simulations (solid curves) and best-fit parameters for the 1-T/2-T models (vertical lines). The lower-temperature of the 2-T model is set by hand to be $T_{\text {peak }}$. The 1-T/2-T model cannot reflect the true temperature distribution of the gas.

difference between the model and the simulation spectra is within a factor of 2. As for the 1-T model, $\Delta$ is noisier for SFR3 as compared to SFR10. From Figure 6, we see that NFD for the 2-T model is nearly same as that for the 1-T model.

In Figure 7 the 2-T model is represented by two vertical solid

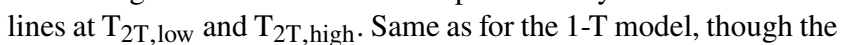
modelled spectra match the simulation spectra well, the temperature distributions from $2 \mathrm{~T}$ models deviate far from the actual distribution. Further, the best-fit $\mathrm{T}_{2 \mathrm{~T}}$, high has little physical meaning.

\section{LOG NORMAL}

In the previous section, we discussed the 1-T and the 2-T models, which assume that all of the mass emitting X-ray has either one or two temperatures. Though such models are able to match the simulation spectra reasonably well, the temperature distributions in these models are not physically motivated. In this Section, we propose a novel temperature model for fitting with the spectra.

As discussed in Section 3.2, the temperature and density distributions in the simulations cover a broad range around a single peak. 
We, therefore, model the temperature distribution with a log-normal distribution, which is expressed mathematically as

$$
p(T)=\frac{1}{\sigma_{T} T \sqrt{2 \pi}} e^{-\frac{\left(\ln \mathrm{T}-\mu_{\mathrm{T}}\right)^{2}}{2 \sigma_{T}^{2}}},
$$

where $\sigma_{T}$ and $\mu_{T}$ are the standard deviation and the mean of the underlying log-normal distribution, respectively.

We generate a "log-normal" box such that the mass distribution in temperature within the box follows a log-normal distribution. To generate a temperature in the log-normal box, we begin with a guess value for $\sigma_{\mathrm{T}}$ and $\mu_{\mathrm{T}}$, and then generate distributions for gas temperature using Equation 1. The density values of the gas in this box also follow a log-normal distribution similar to Equation 1. We generate the spectra and compare it with the simulation spectra. We then adjust the values of these parameters, i.e., $\sigma_{\mathrm{T}}$ and $\mu_{\mathrm{T}}$, to find the best-fit parameters in few iterations with minimized NFD. Once the best-fit temperature value has been obtained, we adjust the mass of gas in the log-normal so as to get the minimum NFD. The best-fit values of $\sigma_{\mathrm{T}}$ and $\mu_{\mathrm{T}}$ are listed in Table 1 .

The fourth and the last panels of Figure 5 show the $\Delta$ between the simulation spectra from SFR3 and SFR10, respectively. We show both the low-resolution (left) and the high-resolution (right) spectra. We note that the log-normal spectra match the simulations spectra well at both high- and low-energy ends. We indicate the NFD values for this model in Figure 6. For both SFR3 and SFR10, these values are lower than the corresponding values for 1-T and 2-T. This indicates that the log-normal model yields a better fit than the 1-T/2-T models.

Figure 8 shows the comparison of the fractional mass distribution. The solid curves indicate the mass distributions from simulation and the dotted curves represent the best-fit for high resolution spectra. The log-normal distribution reproduces the broad distribution in temperature, unlike the vertical delta function used in 1-T/2-T models. The models are able to generate mass fractions at both low and high temperatures. Admittedly, the mass distribution for SFR3 is a better fit than that for SFR10. The reason for this difference in fits is that outflows in SFR10 leave the gas outside of the bi-cone relatively undisturbed; the undisturbed ambient medium contributes the sharp peak at $\sim 2 \times 10^{6} \mathrm{~K}$ to the mass distribution in Figure 8 . We discuss the consequences of bi-polar outflows on spectrum of CGM gas in greater detail in the next Section. That said, the essence of a broad T-distribution is captured for both cases by log-normal model. The fact that the log-normal model can reproduce both the spectra and underlying T-distribution demonstrates its feasibility as a physically-based model, which we argue is better than the 1-T/2-T models.

\section{SPECTRA FROM INSIDE VERSUS OUTSIDE OF OUTFLOW BI-CONES}

From Figure 1 we see that SFR10 has bi-conical outflows resulting different density, temperature and metallicity profiles inside and outside the bi-cones. Interestingly, these features are not obvious in the projected X-ray emission maps (see Figure 3). Here, we investigate whether these outflow features produce visible differences in spectra taken from different azimuthal angles. High angular resolutions of future X-ray missions ( $\sim 5^{\prime \prime}$ in Athena, for example) will allow astronomers to probe CGM at different azimuthal angles of a galaxy and provide information about profiles along and perpendicular to the outflow direction. Keeping this in view, in this Section

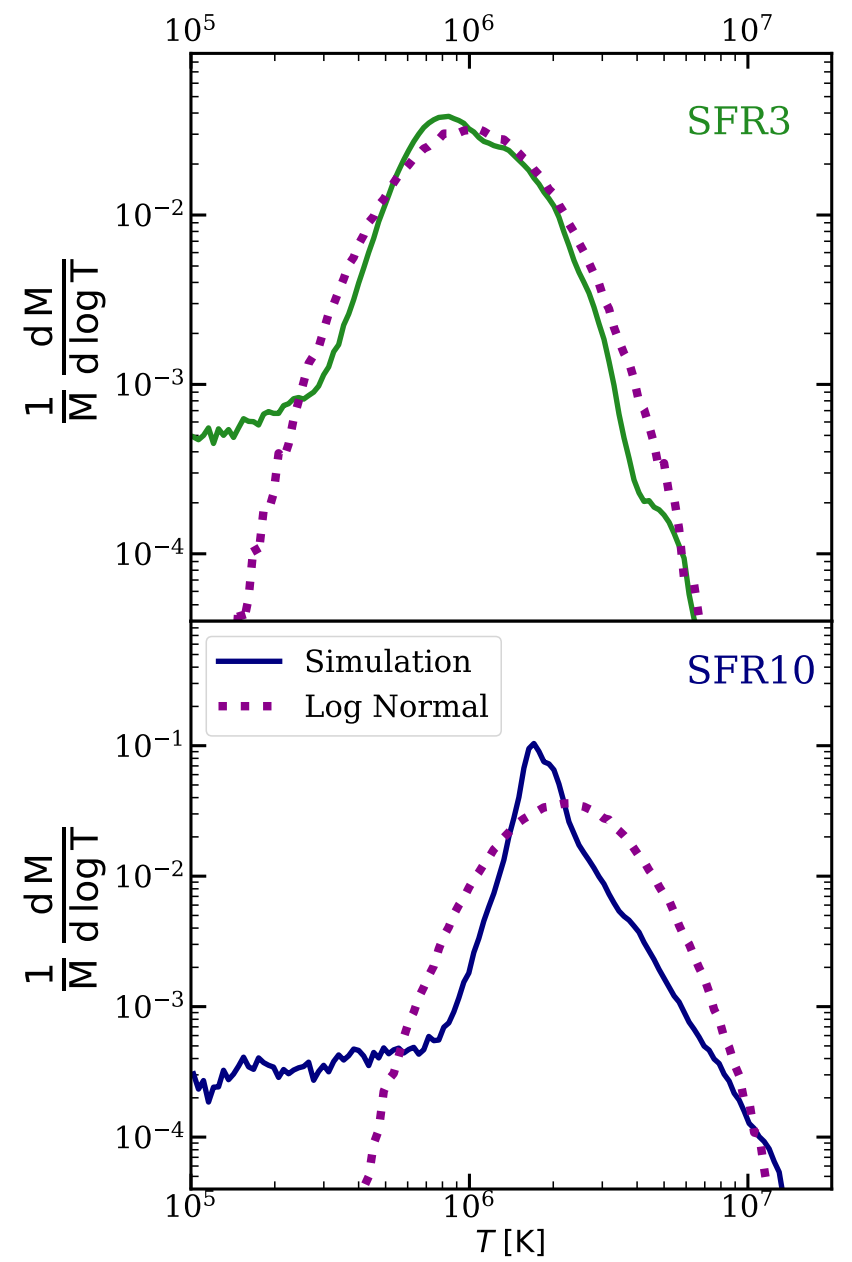

Figure 8. Comparison of the mass distribution of the temperature between simulations (solid curves) and the best-fit log-normal model (dotted curves). The shape of log-normal model better reflects the broad temperature distribution of the gas.

we discuss the gas properties and spectra inside and outside the bi-cones, i.e., the ' $z$ ' and ' $y$ ' axes, respectively.

In Figure 9, we show the variation of density, metallicity and temperature along ' $y$ ' and ' $z$ ' axes as the function of distance from the centre of the galaxy for SFR10. To produce the profiles, we take rectangular boxes along $y$ and $z$ directions with dimensions $[x, y, z] \in[ \pm 10, \pm 400, \pm 10] \mathrm{kpc}$ and $[x, y, z] \in[ \pm 10, \pm 10, \pm 400]$ $\mathrm{kpc}$, centred at the origin of the simulation box. These boxes are denoted as " $y$ " and " $z$ " in the figure. We then average the quantities in the boxes along $y$ - and z-direction, respectively. We note here that all the physical quantities shown here are weighted by $0.5-2.0 \mathrm{keV}$ $\mathrm{X}$-ray luminosity. As expected from Figure 1, the average density, metallicity and temperature decrease away from the centre and are different in the two directions. Along the outflow direction we expect the gas temperature, density and metallicity values to be higher. The starkest difference is seen in the metallicity profiles along the two direction. For " $y$ " direction, the metallicity is relatively flat around $Z_{\odot}$ for the inner $40 \mathrm{kpc}$, whereas for the " $\mathrm{z}$ " direction, it drops fast, to around $0.2 Z_{\odot}$ (metallicity of pre-existing gas) at $40 \mathrm{kpc}$.

Figure 10 shows the fractional mass distribution in temperature along the $z$ - and $y$-directions for $10^{5-7.3} \mathrm{~K}$ gas, in a projected way. To produce these plots, we take rectangular boxes with dimensions 


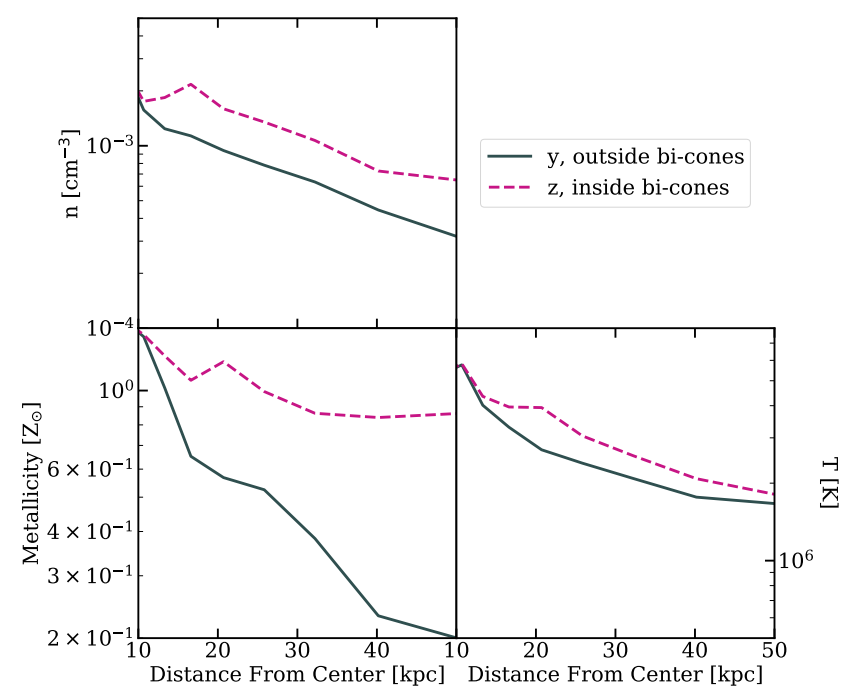

Figure 9. Radial profiles of density, metallicity and temperature along the ' $y$ ' (outside outflow cones) and ' $z$ ' (inside outflow cones) directions for SFR 10. The quantities are weighted by X-ray luminosity $(0.5-2.0 \mathrm{keV})$.

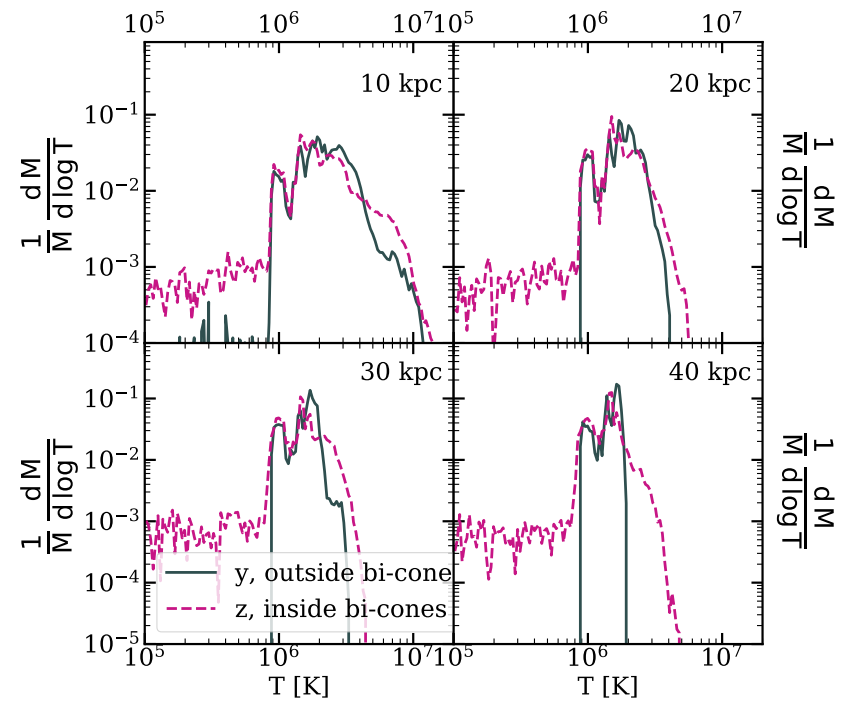

Figure 10. Comparison of temperature distribution in projected boxes $(800 \times$ $10 \times 10 \mathrm{kpc}^{3}$ - the longest edge being along the $x$ axis). The panels show boxes at different distances from the centre of the galaxy, indicated at topright corners. Inside the bi-cones, there is more gas with higher temperature, especially at large distance.

$10 \times 10 \times 800 \mathrm{kpc}$ such that the longest dimension lies along the $x$ axis. We centre the box at different distances from the origin starting at $10 \mathrm{kpc}$. For example, the centre of the box labelled ' $10 \mathrm{kpc}$ ' along $y$ direction is located at $[x, y, z]=[0,10,0] \mathrm{kpc}$. Note that this is different from gas sampling for Figure 9.

The distributions along $y$-direction peak at $\sim 2 \times 10^{6} \mathrm{~K}$ representing the less undisturbed medium which has not been affected by the outflows (other than the shocks). These distributions also become narrower for larger distances from the centre. In the $z$ direction, the high-temperature tail of the mass distribution is prominent, especially at 30 and $40 \mathrm{kpc}$. The asymmetry of gas distribution

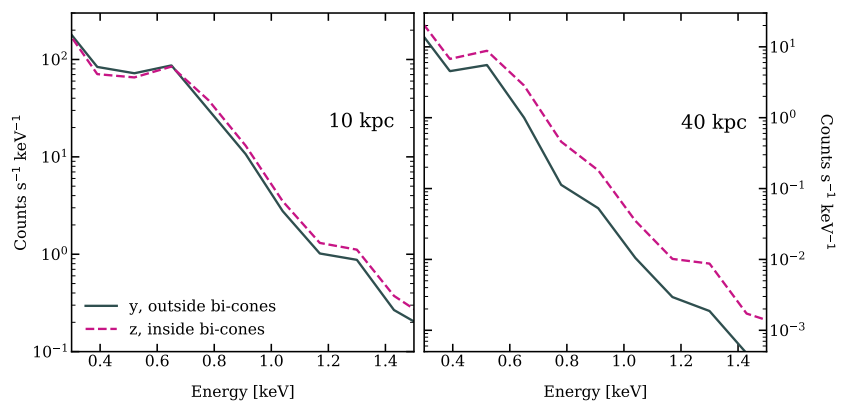

Figure 11. Spectra for boxes along $y$ and $z$ directions, binned at $130 \mathrm{eV}$, located at a distance of 10 and $40 \mathrm{kpc}$, respectively. The spectra are nearly identical at $10 \mathrm{kpc}$, while they differ significantly at $40 \mathrm{kpc}$. The high-energy end is enhanced in the $y$ direction, due to larger amount of high-temperature gas.

in the two directions, generated by the bi-polar outflows, is clearly evident in this Figure.

In Figure 11 we show the spectra arising from boxes at 10 and $40 \mathrm{kpc}$. For producing the spectra, we use the projection of the boxes used for Figure 10 along the $x$ axis, with arbitrary normalisations. We show only the low-resolution spectra binned at $130 \mathrm{eV}$. At 10 $\mathrm{kpc}$ the spectra from the two directions are nearly identical. This is not surprising as from Figure 10, the temperature distribution for Xray emitting gas is nearly same at this distance from the centre. The difference in spectra is more prominent at $40 \mathrm{kpc}$, especially at the high energy end. The spectra are less steep for the $z$-direction. This is because the $z$-direction has hotter gas at $40 \mathrm{kpc}$ than $y$-direction, as seen from Figure 10.

\section{DISCUSSION}

\subsection{Mock Observations for Chandra, Athena, Lynx, HUBS \& XRISM}

In this Section, we discuss mock spectra produced from the simulation data. For our discussion, we focus on the following four instruments: ACIS-S on Chandra (Weisskopf et al. 2000), X-Ray Integral Field Unit on Athena (Barcons et al. 2015), X-ray Microcalorimeter (Main Array) on Lynx (Gaskin et al. 2019), the Hot Universe Baryon Surveyor (HUBS) (Cui et al. 2020) and the X-ray Imaging and Spectroscopy Mission (XRISM) (XRISM Science Team 2020). Except for Chandra, the rest of the telescopes are planned mission with enhanced capabilities (Li 2020, see for a comparison). These planned mission have a much higher energy resolution and sensitivity which will enable much more accurate measurements of the metallicity, gas kinematics, etc.

We use a python-based module, $\mathrm{SOXS}^{6}$, to create a photon list from the simulation data set assuming a distance of $25 \mathrm{Mpc}$ and an effective area of $2.5 \mathrm{~m}^{2}$. We choose this distance to ensure that the inner region of the CGM ( $\lesssim 20 \mathrm{kpc}$ ), which emits most strongly (see Figure 3), fits within the field of view $\left(\sim 5^{\prime}\right)$ of Athena and $\operatorname{Lynx}^{7}$. A subset of photons from the list are convolved with the instrument response in order to produce the spectra for an exposure time of $2 \mathrm{Ms}$ for Chandra and Athena and Lynx, and 7 and $5 \mathrm{Ms}$ for

\footnotetext{
${ }^{6}$ http://hea-www.cfa.harvard.edu/soxs/.

7 Chandra and HUBS have larger fields of view, $20^{\prime}$ and $1^{\circ}$, respectively, while XRISM has a comparable field of view, $2.9^{\prime}$.
} 


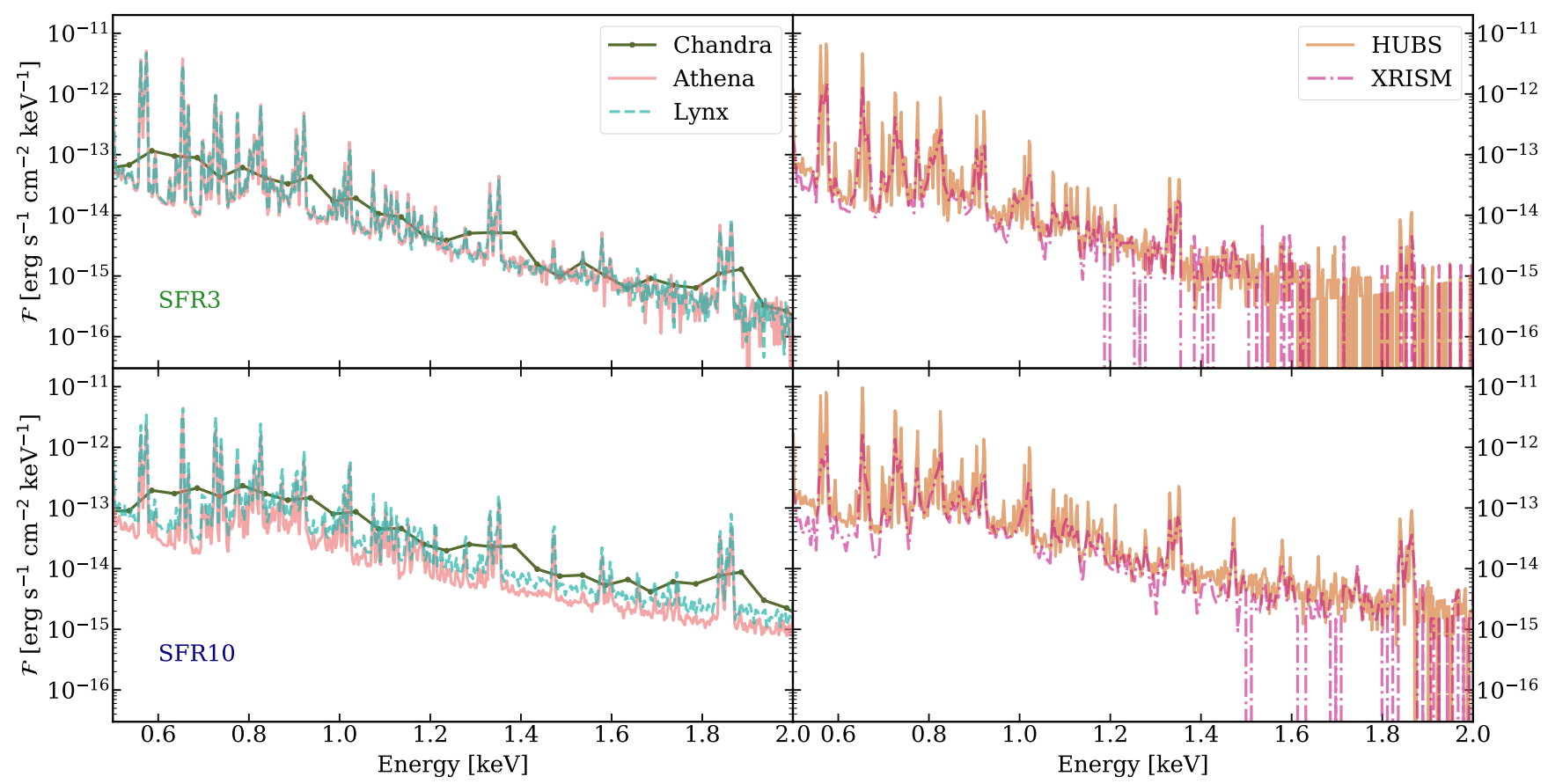

Figure 12. Mock spectra for Chandra (green), Athena (coral), Lynx (green), HUBS (yellow) and XRISM (magneta) spectra for SFR3 (top) and SFR10 (bottom). These spectra do not include background or foreground. The numbers on the top right corner indicate the $0.5-2.0 \mathrm{keV}$ luminosity in units of erg s ${ }^{-1}$ calculated from these spectra. The spectral resolution of these instruments are 50.0 (Chandra), 2.5 (Athena), 3.0 (Lynx) and 2.0 (HUBS) eV.

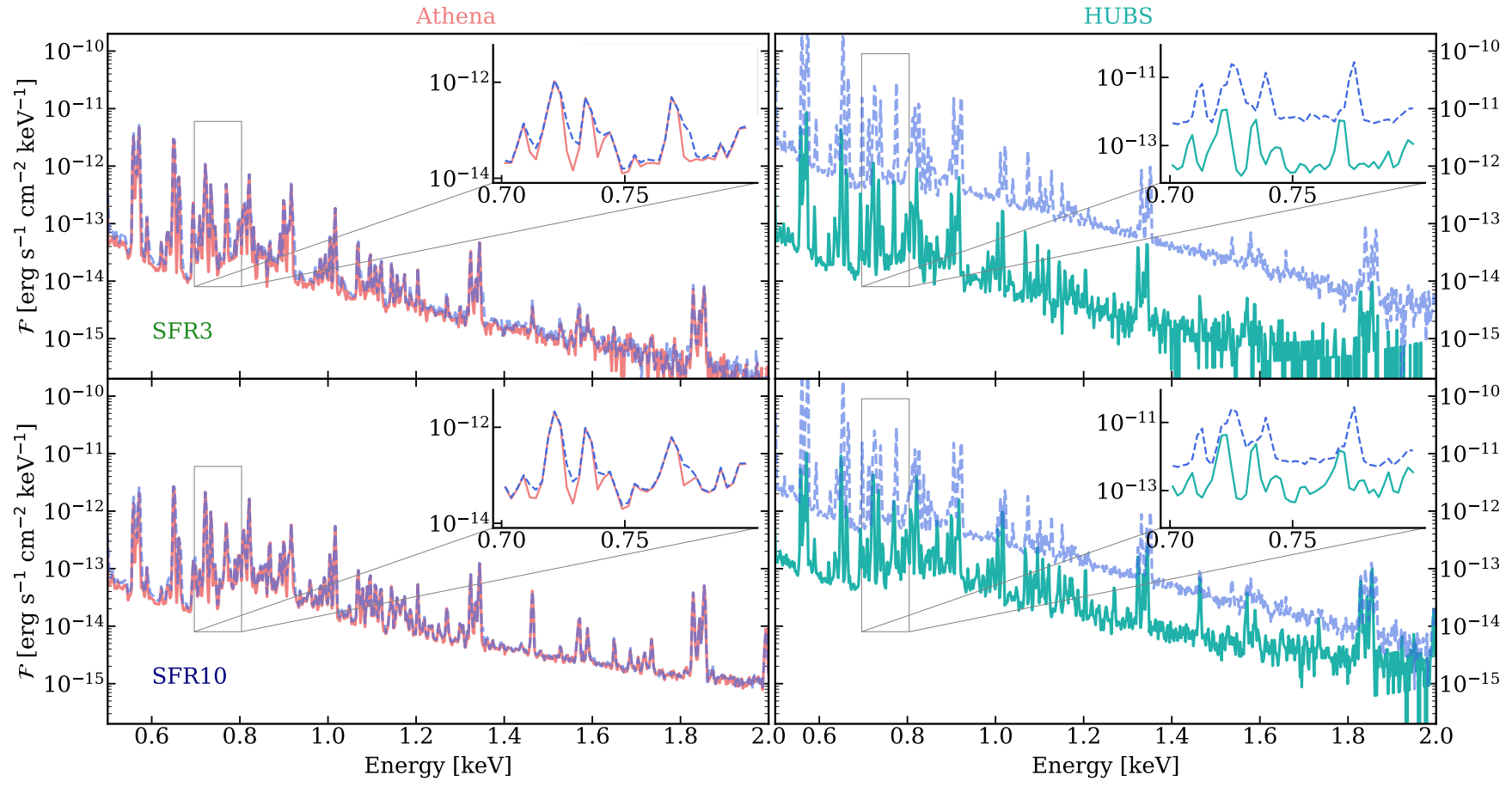

Figure 13. Mock spectra from Athena (left) and HUBS (right) for SFR3 and SFR10 at a distance of $25 \mathrm{Mpc}$. We show the effect of adding Galactic foreground to the spectra in dashed lines. The spectra from HUBS exhibit a higher contribution from the foreground because of its large field of view $\left(\sim 1^{\circ}\right)$, as compared to $5.9^{\prime}$ field of view of Athena. In the top-right corner of each panel, we show the spectra zoomed-in between 0.7 and $0.8 \mathrm{keV}$. Emission lines at a distance of $25 \mathrm{Mpc}$ are not blended into the foreground for these eV-level instruments. 
HUBS and XRISM, respectively. We choose the exposure time for an instrument to minimise noise from the resulting spectra while ensuring that the photon list generated is not over-sampled. We take the RA and Dec as $142.8^{\circ}$ and $+84.2^{\circ}$, respectively. The choice of the RA and Dec is arbitrary but it so happens that these coordinates correspond to those of NGC 4631. We stress that our intention is not to compare the simulation spectra with this particular galaxy. . For Chandra, the response files are for Cycle 22. For Galactic absorption, we set the column density as $1.93 \times 10^{20} \mathrm{~cm}^{-2}$.

In Figure 12, we show the flux obtained from each telescope for SFR3 (top) and SFR10 (bottom) between $0.5-2.0 \mathrm{keV}$. Galactic foreground is not included. We note that Athena, Lynx, HUBS and XRISM, having a spectral resolution of 2.5, 3.0, 2.0 and $5 \mathrm{eV}$, respectively, are able to resolve line features currently unachievable by Chandra. All the three planned instruments are able to produce consistent spectra. The spectra generated using HUBS and XRISM are noisier than those from the other instruments. The shape of the spectra from the three high-resolution instruments are comparable. We note here that because of their smaller field of view, the focus of Athena and Lynx will be on the smaller regions of galactic winds. XRISM will be able to resolve individual spectral lines, enabling observers to probe the multiphase structure of CGM gas (XRISM Science Team 2020).

Figure 13 shows the spectra from SFR3 (top) and SFR10 (bottom) for Athena and HUBS, adding Galactic foreground. The galaxy is placed at a distance of $25 \mathrm{Mpc}$. We use SOXS to add the Galactic foreground, which is modeled as a uniform and diffuse plasma taken to be the sum of two thermal models at $\mathrm{kT}=0.2$ and $0.099 \mathrm{keV}$. The total foreground flux of an instrument depends on its field of view (FOV). As a result, the foreground flux of HUBS (FOV $\sim 1^{\circ}$ ) is much larger than that added to Athena (FOV $\sim 5^{\prime}$ ). For HUBS, in the softer energy band, the foreground is $\sim 15$ times larger than the signal itself. In the top-right corner of each panel in Figure 13, we also show the zoom-in spectra between $0.7-0.8 \mathrm{keV}$. There we can see that the lines from the external CGM are redshifted relative to the foreground. This indicates that at the eV-level spectral resolution, the emission lines from the CGM at this relatively close distance $(25 \mathrm{Mpc})$ are not blended into the foreground.

\subsection{Connection to Other Works}

The 1-T/2-T model have been used to fit the spectra of many nearby star-forming galaxies (Dahlem et al. 1998; Tüllmann et al. 2006; Yamasaki et al. 2009; Owen \& Warwick 2009; Mineo et al. 2012; Bogdán et al. 2013a; Lopez et al. 2020). Our best-fit temperatures are broadly consistent with the reported values. For MW, a hot component has been detected close to the virial temperature of the halo, $\sim(2-3) \times 10^{6} \mathrm{~K}$, in emission (Henley \& Shelton 2013; Nakashima et al. 2018) as well as absorption Hagihara et al. (2010); Gupta et al. (2012); Fang et al. (2015); Gupta et al. (2017); Das et al. (2019).

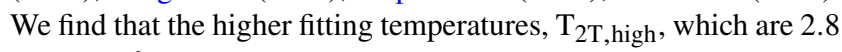
and $3 \times 10^{6}$ Krespectively for SFR 3 and SFR10, are consistent with the observations. That said, as we emphasize in previous sections, the best-fit temperatures using 1-T/2-T models may not reflect faithfully the underlying temperature distribution. Theoretically, it may be possible to find several mathematical functions, including 3-T model (Lopez et al. 2020), that fit the spectra even better than the proposed log-normal. However, not all of these functions will be able faithfully reflect the temperature distribution of the hot CGM, which from the simulations we know is close to a log-normal.

To illustrate the applicability of the log-normal model to ex-

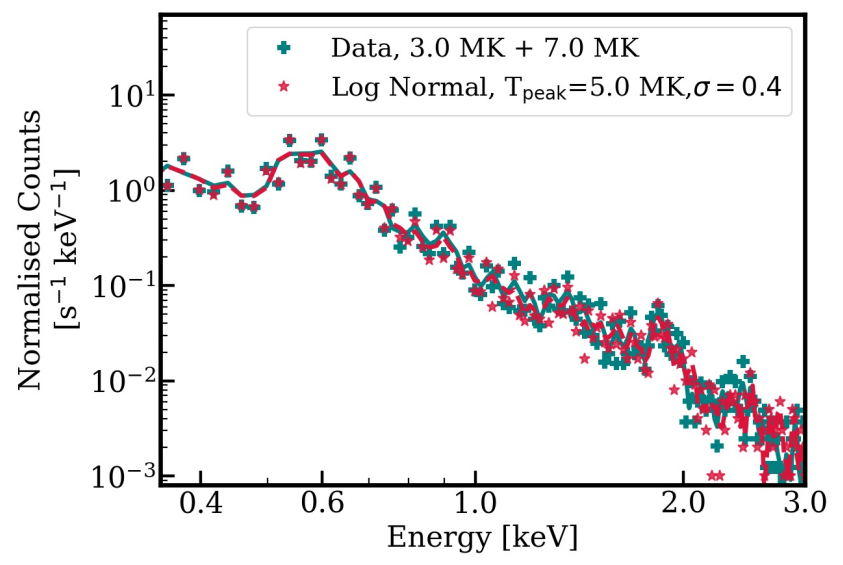

Figure 14. The spectrum for NGC 4631 using fitting parameters given in $\mathrm{Li}$ \& Wang (2013) compared with a log-normal box. We reproduce the Chandra spectrum for NGC 4631 from Li \& Wang (2013) (teal) by superposing normalised spectra from two uniform temperature boxes at $3 \times 10^{6}$ and $7 \times 10^{6} \mathrm{~K}$, which are obtained by $2 \mathrm{~T}$ model fitting reported by Li \& Wang (2013). In green we show the best-fit spectrum from the log-normal model with a peak at $5 \times 10^{6} \mathrm{~K}$. The symbols represent the mock data generated using SOXS, while the lines represent a fit to the data points.

isting data, we discuss the Chandra spectrum of NGC 4631. Wang et al. (2001) and Li \& Wang (2013) discuss the Chandra spectrum for NGC 4631 and fit it using the 2 T model at $\sim 3 \times 10^{6}$ and $\sim 7 \times 10^{6}$ $\mathrm{K}$, having luminosities equal to $9.3 \times 10^{38}$ and $13.7 \times 10^{38} \mathrm{erg} \mathrm{s}^{-1}$, respectively. Figure 3 of Wang et al. (2001) shows that the $2 \mathrm{~T}$ model can fit the data quite well.

We reproduce the spectrum using these temperatures in Figure 14 (teal solid line) following the procedure detailed in Section 3.4. We convolve this spectrum with Chandra instrument response corresponding to Cycle 0 to generate a 60 ks observation, same as that used for the actual observation in Li \& Wang (2013). We take the values for RA and Dec, the column density, and the distance estimates from Table 1 of Li \& Wang (2013).

In order to compare with the log-normal model, construct lognormal temperature box by assuming a peak temperature and a width of the log-normal distribution curve. We produce the Chandra mock spectrum from such a distribution for $60 \mathrm{ks}$ observation period and the relevant parameters for NGC 4631. We find that the best-fit peak temperature is $5 \times 10^{6} \mathrm{~K}$ with a corresponding $\sigma=0.4$, and we show the best-fit log-normal spectrum in Figure 14 (red dashed line). We note that log-normal spectrum matches the observed spectrum well. This shows that log-normal model is able to fit the existing observations as well.

Observations also indicate that models beyond a simple 1$\mathrm{T} / 2-\mathrm{T}$ is required. For example, broad distributions of temperature have been used to explain the emission (Strickland et al. 2002) and absorption studies (Gissis et al. 2020) where multiple ions and ionization states are present. Spectra from galaxy clusters also has been interpreted using multi-temperature fits (Sanders et al. 2010; Werner et al. 2013; Hitomi Collaboration et al. 2018). The lognormal temperature model has also been used in other context for fitting X-ray spectra. For example, (Ge et al. 2015) model the X-ray emission from unresolved stellar contributions using a log-normal distribution. Gaussian temperature distributions have been used previously for fitting X-ray spectra from clusters (Kaastra et al. 2004; Simionescu et al. 2009). In the context of groups and clusters, we 
do expect that similar physical processes, which determine the lognormal shape of the temperature distribution of the gas in our simulations, will be operating and can leave the underlying temperature and density distributions broadened. The high spectral resolution (about $49 \mathrm{eV}$ at $0.3 \mathrm{keV}$ ) of the eROSITA could potentially detect these distributions in its targets (Predehl et al. 2021).

Log-normal distribution has been discussed in recent theoretical studies of CGM as well. Several simulations, evolving the CGM both in the cosmological context and in an isolated environment, found gas temperature and density have a broad distribution at different radii (Lochhaas et al. 2020, 2021; Fielding et al. 2020). Physically, a broadened distribution in temperature can arise because of several factors, such as the interaction of enriched gas with the ambient medium, adiabatic expansion of hot outflows, the interaction of outflows from multiple star formation events, radiative cooling, turbulent motion, etc (Blaisdell et al. 1993). Further, gas models obeying log-normal distributions are also used to explain the observations of emission and column densities of oxygen lines (Faerman et al. 2017; McQuinn \& Werk 2018; Voit 2019; Faerman et al. 2020).

\section{SUMMARY}

Hot galactic outflows emerge as a result of star formation. They interact with the ambient medium and produce multi-phase gas in the CGM of the galaxy. The outflows from SFR3 form a large-scale fountain and nearly isotropic CGM, while those in SFR10 expand bi-conically along the vertical direction.

The main conclusions of the Paper are the following:

(i) The X-ray emitting CGM exhibits a broad range of temperatures, density and metallicity (Figure 1,2).

(ii) The broad temperature range of the X-ray emitting CGM cannot be characterised using 1-T/2-T models, even though such models may fit the X-ray emission spectra (Figure 5, 7).

(iii) The mass distribution of X-ray emitting CGM can be better approximated by a log-normal distribution in temperature for both low and high SFR. Such a distribution can be described using a two parameters, i.e, a peak temperature and a width around the peak (Eq. 1).

(iv) The log-normal distribution fits better the spectra than 1T/2-T model, and best-fit parameters reproduce the underlying temperature distribution (Figure 5, 6, 8).

(v) In the high SFR case, SFR10, the outflows introduce an asymmetry in gas properties inside and outside of the outflow bicones. This difference is reflected in the X-ray spectra, as gas inside the cone is hotter and more metal-enriched (Figure 9, 10, 11).

(vi) Future X-ray telescopes with $\sim \mathrm{eV}$ resolution, such as Athena, Lynx and HUBS, will be able resolve the line features, providing much more details about the hot CGM (Figure 12,13).

\section{ACKNOWLEDGEMENTS}

We dedicate this paper to all the frontline workers across the world, who have worked so hard to keep us safe during the challenging time of COVID-19. We thank the anonymous referee for their useful comments. ML thanks the beneficial discussion with S. Mathur, J-T Li, D. Wang, S. Das, and L. Lopez. We thank the KITP-Halo21 program (supported by NSF grant PHY-1748958) for stimulating scientific discussions. Data analysis and visualization are partly done using the yt project (Turk et al. 2011). Part of the computation/analysis are performed on the Rusty cluster of the Simons Foundation. We thank the Scientific Computing Core of the Simons Foundation for their technical support. A part of the analysis was also done at SahasraT cluster, maintained by the Super-computer Education and Research Centre, Indian Institute of Science, and the cluster at Shanghai Astronomical Observatory. AV would like to thank the staff at both these institutes for their support.

\section{DATA AVAILABILITY}

The data underlying this article will be shared on reasonable request to the corresponding author.

\section{REFERENCES}

Anderson M. E., Bregman J. N., 2011, ApJ, 737, 22

Anderson M. E., Churazov E., Bregman J. N., 2016, MNRAS, 455, 227

Armillotta L., Fraternali F., Werk J. K., Prochaska J. X., Marinacci F., 2017, MNRAS, 470, 114

Barcons X., Nandra K., Barret D., den Herder J. W., Fabian A. C., Piro L., Watson M. G., the Athena Team 2015, in Journal of Physics Conference Series. p. 012008, doi:10.1088/1742-6596/610/1/012008

Biffi V., Dolag K., Böhringer H., Lemson G., 2012, MNRAS, 420, 3545

Biffi V., Dolag K., Böhringer H., 2013, MNRAS, 428, 1395

Blaisdell G. A., Mansour N. N., Reynolds W. C., 1993, Journal of Fluid Mechanics, 256, 443

Bogdán Á., Forman W. R., Kraft R. P., Jones C., 2013a, ApJ, 772, 98

Bogdán Á., Forman W. R., Kraft R. P., Jones C., 2013b, ApJ, 772, 98

Bogdán Á., Bourdin H., Forman W. R., Kraft R. P., Vogelsberger M., Hernquist L., Springel V., 2017, ApJ, 850, 98

Burkert A., 1995, ApJ, 447, L25

Cui W., et al., 2020, in Society of Photo-Optical Instrumentation Engineers (SPIE) Conference Series. p. 114442S (arXiv:2101.05587), doi:10.1117/12.2560871

Dahlem M., Weaver K. A., Heckman T. M., 1998, ApJS, 118, 401

Dai X., Anderson M. E., Bregman J. N., Miller J. M., 2012, ApJ, 755, 107

Das S., Mathur S., Nicastro F., Krongold Y., 2019, ApJ, 882, L23

Davies J. J., Crain R. A., Oppenheimer B. D., Schaye J., 2020, MNRAS, 491, 4462

Dekel A., et al., 2009, Nature, 457, 451

Fabian A. C., 2010, Cosmic Feedback from AGN (arXiv:0912.0880), doi:10.1017/S1743921310006691

Faerman Y., Sternberg A., McKee C. F., 2017, ApJ, 835, 52

Faerman Y., Sternberg A., McKee C. F., 2020, ApJ, 893, 82

Fang T., Buote D., Bullock J., Ma R., 2015, ApJS, 217, 21

Fielding D. B., Bryan G. L., 2021, arXiv e-prints, p. arXiv:2108.05355

Fielding D., Quataert E., Martizzi D., Faucher-Giguère C.-A., 2017, MNRAS, 470, L39

Fielding D. B., et al., 2020, ApJ, 903, 32

Ford A. B., Davé R., Oppenheimer B. D., Katz N., Kollmeier J. A., Thompson R., Weinberg D. H., 2014, MNRAS, 444, 1260

Forman W., Jones C., Tucker W., 1985, ApJ, 293, 102

Gaskin J. A., et al., 2019, Journal of Astronomical Telescopes, Instruments, and Systems, 5, 021001

Ge C., et al., 2015, ApJ, 812, 130

Gissis I., Peretz U., Behar E., 2020, MNRAS, 492, 5634

Grimes J. P., Heckman T., Strickland D., Ptak A., 2005, ApJ, 628, 187

Gupta A., Mathur S., Krongold Y., Nicastro F., Galeazzi M., 2012, ApJ, 756, L8

Gupta A., Mathur S., Krongold Y., 2017, ApJ, 836, 243

Hagihara T., Yao Y., Yamasaki N. Y., Mitsuda K., Wang Q. D., Takei Y., Yoshino T., McCammon D., 2010, PASJ, 62, 723

Henley D. B., Shelton R. L., 2013, ApJ, 773, 92

Hernquist L., 1993, ApJS, 86, 389 
Hitomi Collaboration et al., 2018, PASJ, 70, 11

Hummels C. B., Bryan G. L., Smith B. D., Turk M. J., 2013, MNRAS, 430, 1548

Kaastra J. S., et al., 2004, A\&A, 413, 415

Kereš D., Katz N., Weinberg D. H., Davé R., 2005, MNRAS, 363, 2

Kim C.-G., Ostriker E. C., Raileanu R., 2017, ApJ, 834, 25

Lehner N., Howk J. C., Thom C., Fox A. J., Tumlinson J., Tripp T. M., Meiring J. D., 2012, MNRAS, 424, 2896

Lehner N., et al., 2013, ApJ, 770, 138

Li J.-T., 2020, Astronomische Nachrichten, 341, 177

Li M., Bryan G. L., 2020, ApJ, 890, L30

Li M., Tonnesen S., 2020, ApJ, 898, 148

Li J.-T., Wang Q. D., 2013, MNRAS, 435, 3071

Li J.-T., Bregman J. N., Wang Q. D., Crain R. A., Anderson M. E., Zhang S., 2017a, ApJS, 233, 20

Li M., Bryan G. L., Ostriker J. P., 2017b, ApJ, 841, 101

Lochhaas C., Bryan G. L., Li Y., Li M., Fielding D., 2020, MNRAS, 493, 1461

Lochhaas C., Tumlinson J., O’Shea B. W., Peeples M. S., Smith B. D., Werk J. K., Augustin R., Simons R. C., 2021, arXiv e-prints, p. arXiv:2102.08393

Lopez L. A., Mathur S., Nguyen D. D., Thompson T. A., Olivier G. M., 2020, arXiv e-prints, p. arXiv:2006.08623

McKee C. F., Ostriker J. P., 1977, ApJ, 218, 148

McQuinn M., Werk J. K., 2018, ApJ, 852, 33

Mineo S., Gilfanov M., Sunyaev R., 2012, MNRAS, 426, 1870

Miyamoto M., Nagai R., 1975, PASJ, 27, 533

Morrison R., McCammon D., 1983, ApJ, 270, 119

Mulchaey J. S., Jeltema T. E., 2010, ApJ, 715, L1

Nakashima S., Inoue Y., Yamasaki N., Sofue Y., Kataoka J., Sakai K., 2018, ApJ, 862, 34

Nguyen D. D., Thompson T. A., 2021, MNRAS, 508, 5310

O'Sullivan E., Forbes D. A., Ponman T. J., 2001, MNRAS, 328, 461

Oppenheimer B. D., Davé R., 2008, MNRAS, 387, 577

Owen R. A., Warwick R. S., 2009, MNRAS, 394, 1741

Predehl P., et al., 2021, A\&A, 647, A1

Sanders J. S., Fabian A. C., Frank K. A., Peterson J. R., Russell H. R., 2010, MNRAS, 402, 127

Sarkar K. C., Nath B. B., Sharma P., Shchekinov Y., 2015, MNRAS, 448, 328

Silk J., 1977, ApJ, 211, 638

Simionescu A., Werner N., Böhringer H., Kaastra J. S., Finoguenov A., Brüggen M., Nulsen P. E. J., 2009, A\&A, 493, 409

Smith B. D., et al., 2017, MNRAS, 466, 2217

Strickland D. K., Heckman T. M., Weaver K. A., Hoopes C. G., Dahlem M., 2002, ApJ, 568, 689

Strickland D. K., Heckman T. M., Colbert E. J. M., Hoopes C. G., Weaver K. A., 2004, ApJ, 606, 829

Suchkov A. A., Berman V. G., Heckman T. M., Balsara D. S., 1996, ApJ, 463,528

Suresh J., Rubin K. H. R., Kannan R., Werk J. K., Hernquist L., Vogelsberger M., 2017, MNRAS, 465, 2966

Tüllmann R., Pietsch W., Rossa J., Breitschwerdt D., Dettmar R. J., 2006, A\&A, 448, 43

Turk M. J., Smith B. D., Oishi J. S., Skory S., Skillman S. W., Abel T., Norman M. L., 2011, ApJS, 192, 9

Vijayan A., Sarkar K. C., Nath B. B., Sharma P., Shchekinov Y., 2018, MNRAS, 475, 5513

Voit G. M., 2019, ApJ, 880, 139

Wang Q. D., Immler S., Walterbos R., Lauroesch J. T., Breitschwerdt D., 2001, ApJ, 555, L99

Wang Q. D., Li J., Jiang X., Fang T., 2016, MNRAS, 457, 1385

Weisskopf M. C., Tananbaum H. D., Van Speybroeck L. P., O’Dell S. L., 2000, in Truemper J. E., Aschenbach B., eds, Society of Photo-Optical Instrumentation Engineers (SPIE) Conference Series Vol. 4012, XRay Optics, Instruments, and Missions III. pp 2-16 (arXiv:astroph/0004127), doi:10.1117/12.391545

Werner N., et al., 2013, ApJ, 767, 153

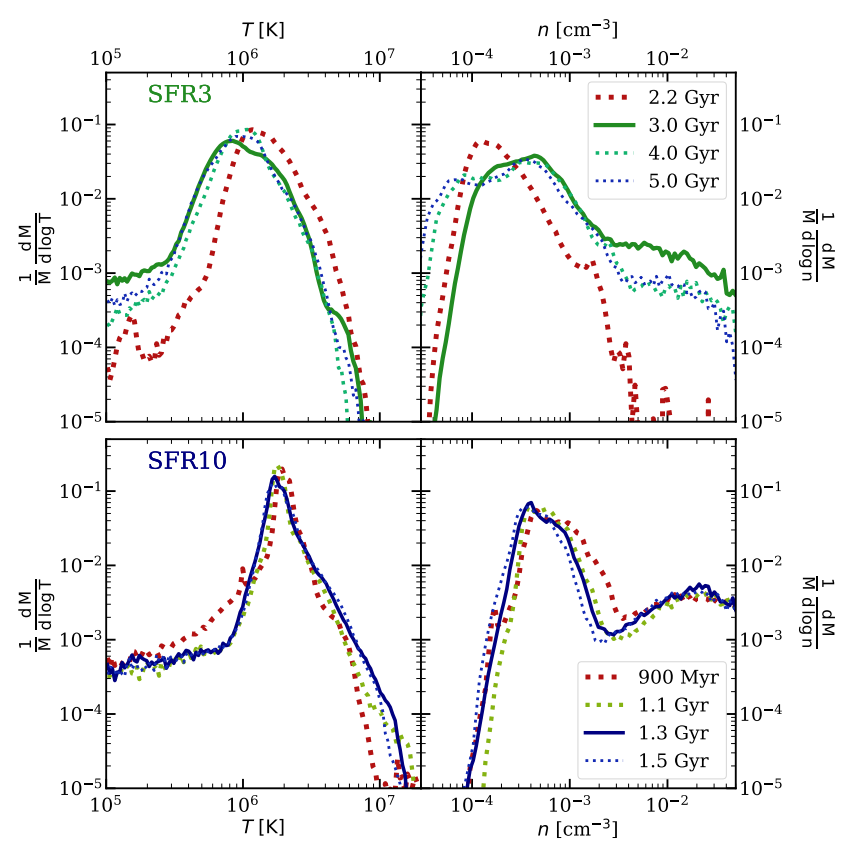

Figure A1. Mass probability distribution for logarithmic density and temperature for SFR3 (top) and SFR10 (bottom). Each curve represents a different time step in the simulations. The systems reach a quasi steady state in terms of mass distribution. Maximum mass is at $\sim 10^{6} \mathrm{~K}$ for all the times. The solid green and blue curves represent the time steps analysed in the main body of the paper.

XRISM Science Team 2020, arXiv e-prints, p. arXiv:2003.04962 Yamasaki N. Y., Sato K., Mitsuishi I., Ohashi T., 2009, PASJ, 61, S291

\section{APPENDIX A: TEMPORAL VARIATIONS IN THE MASS DISTRIBUTION}

In previous Sections, we discuss the results from one snapshot of the simulation runs. However, the underlying density and temperature distributions of the gas does not vary significantly over time after the system reaches a steady state.

In Figure A1, we show 1-D mass plots for different time steps for SFR3 and SFR10. The solid curves, at 3 Gyr for SFR3 and 1.3 Gyr for SFR10, are identical to the ones analysed in the main body of the paper. We note that in the SFR3 case, condensation of cool gas begins in the CGM at about $2.2-3 \mathrm{Gyr}$ and the system settles into a quasi-steady state thereafter. For the SFR10case the hot CGM attains a quasi-steady state (for a few 100s Myr) after $700 \mathrm{Myr}$. The variations in the density and temperature profiles that we see in Figure A1 produce only a factor of 2 variation in the spectra, as shown in Figure 5.

\section{APPENDIX B: VARIATIONS IN SIZES OF SELECTED CGM}

Our analysis is limited to the inner $R \lesssim 50 \mathrm{kpc}$ of the simulation domain, which we refer to as the "region of interest". We check how varying the size of the included region change our results, including both the inner and outer boundaries.

Figure B1 shows the fractional mass distribution of temperature (left) and density (right) for SFR3 (top) and SFR10 (bottom). This figure is identical to Figure A1, except that now we show the 


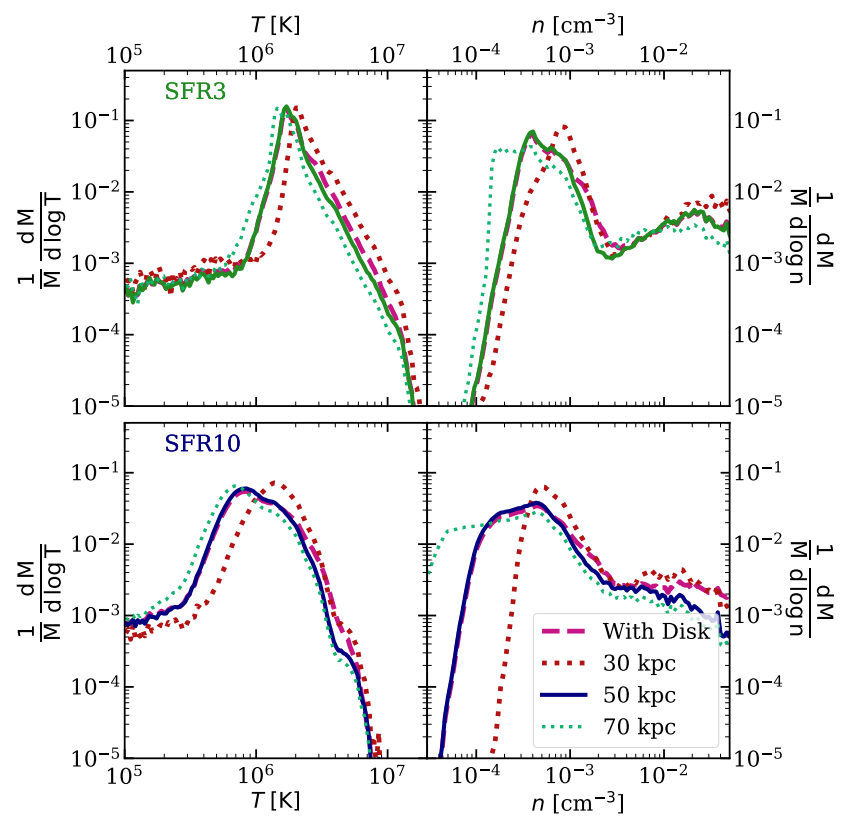

Figure B1. Mass probability distribution for logarithmic density and temperature for SFR3 (top) and SFR10 (bottom). Each curve represents a different size of selected CGM whose dimension is indicated at the bottom right corner of the figure. The curve corresponding to "With Disk" refers to a $50 \mathrm{kpc}$ region which included the disk. Mass distributions do not vary much when changing the size of the selected region.

fractional mass distribution when we consider regions of different sizes. We show the outer radii of 30,50 (fiducial value) and 70 $\mathrm{kpc}$, respectively. In all these cases we have removed the disk region $\left(\pi 20 \times 20 \times( \pm 3) \mathrm{kpc}^{3}\right)$ from the analysis using the procedure described in Section 3.2. The distributions are nearly identical. We also show the fractional mass distribution for a region that includes the disk region in each panel, i.e. all gas within the outer boundary (dashed curve). The fractional mass distribution does not change significantly. The differences in fractional mass distribution of density of different sizes are noticeable, especially between 30 and 70 $\mathrm{kpc}$. This difference arises because large regions include relatively higher proportion of low density gas. The regions with low density regions do contribute significantly to the X-ray emission because of smaller density and because they lie at large radii, far away from the region of interest.

Figure B2 shows the low-resolution spectra corresponding to each of the different box sizes, which do not show significant variation, either. This is not surprising since the underlying mass distribution does not vary significantly by changing the box size. Thus, we conclude that our results are not sensitive to the selected region of interest

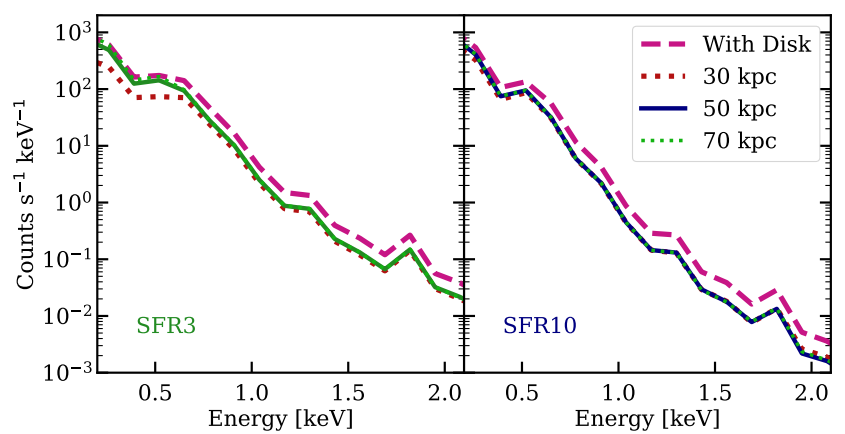

Figure B2. Low-resolution spectra for SFR3 (left) and SFR10 (right). Each curve represents a different box size whose dimension is indicated at the bottom right corner of the figure. The solid curves of the same color represent those in Figure 2. The variation of the spectra with different sizes of selected regions is minor. 